\title{
Large-scale Invasion Percolation with Trapping for Upscaling Capillary-Controlled Darcy-scale Flow
}

\author{
Hasan A. Nooruddin ${ }^{1}$ • Martin J. Blunt ${ }^{1}$
}

Received: 28 July 2017 / Accepted: 26 October 2017 / Published online: 28 November 2017

(C) The Author(s) 2017. This article is an open access publication

\begin{abstract}
We apply steady-state capillary-controlled upscaling in heterogeneous environments. A phase may fail to form a connected path across a given domain at capillary equilibrium. Moreover, even if a continuous saturation path exists, some regions of the domain may produce disconnected clusters that do not contribute to the overall connectivity of the system. In such cases, conventional upscaling processes might not be accurate since identification and removal of these isolated clusters are extremely important to the global connectivity of the system and the stability of the numerical solvers. In this study, we address the impact of percolation during capillary-controlled displacements in heterogeneous porous media and present a comprehensive investigation using random absolute permeability fields, for water-wet, oil-wet and mixed-wet systems, where J-function scaling is used to relate capillary pressure, porosity and absolute permeabilities in each grid cell. Important information is revealed about the average connectivity of the phases and trapping at the Darcy scale due to capillary forces. We show that in oil-wet and mixed-wet media, large-scale trapping of oil controlled by variations in local capillary pressure may be more significant than the local trapping, controlled by pore-scale displacement.
\end{abstract}

Keywords Immiscible displacements - Capillary-driven flow · Large-scale percolation · Steady-state upscaling

\section{List of symbols}

$A_{\mathbf{p}} \quad$ Symmetric matrix used in percolation solver for a given phase

$A_{i j} \quad$ Cross-sectional area between two nodes $\left(\mathrm{m}^{2}\right)$

$\boldsymbol{B} \quad$ Adjacent matrix of a simulation model

Hasan A. Nooruddin

h.nourdeen14@imperial.ac.uk

Martin J. Blunt

m.blunt@imperial.ac.uk

1 Department of Earth Science and Engineering, Imperial College London, London, UK 


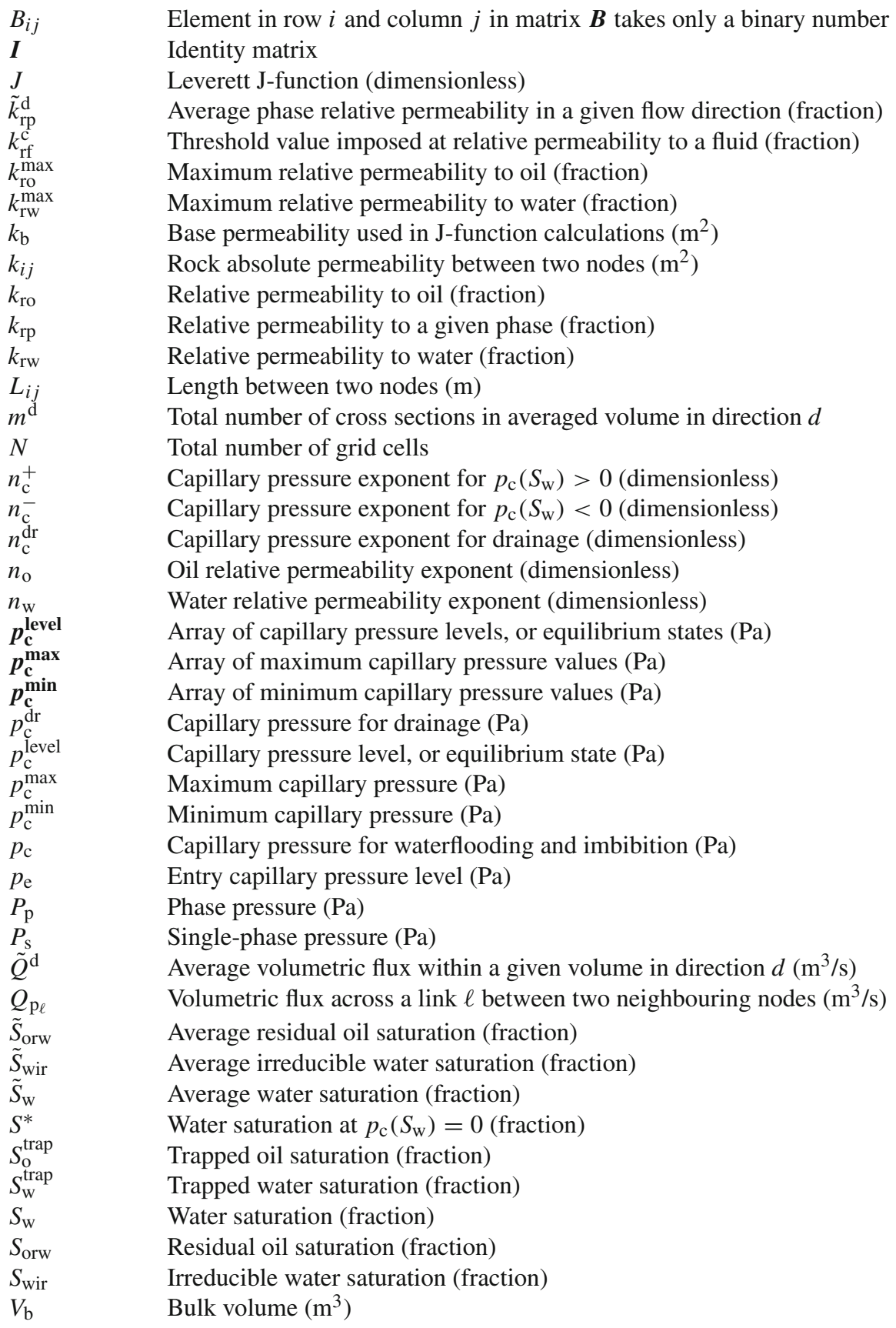

\section{Acronyms}

$\begin{array}{ll}\text { MW } & \text { Mixed-wet } \\ \text { OW } & \text { Oil-wet }\end{array}$


SWW Strongly water-wet

WWW Weakly water-wet

\section{Greek Symbols}

$\eta_{\mathrm{p}}$

$\gamma_{\mathrm{p}}$

$\boldsymbol{\Gamma}_{\mathrm{p}}$

$\gamma_{\mathbf{p}}$

$\lambda_{\mathrm{p}}$

$\lambda_{\mathrm{s}}$

$\mu_{k}$

$\mu_{\mathrm{p}}$

$\mu_{\mathrm{s}}$

$\mu_{\ln k}$

$\Omega_{J}$

$\phi$

$\phi_{\mathrm{b}}$

$\psi_{\mathrm{p}}^{\infty}$

$\psi_{\mathrm{p}}$

$\psi_{\mathrm{p}}^{\infty}$

$\psi_{\mathbf{p}}^{0}$

$\psi_{\mathbf{p}}^{k+1}$

$\psi_{\mathrm{p}}$

$\sigma_{\ln k}$

$\sigma_{\text {wo }}$

$\theta_{\text {wo }}$

$\xi_{\mathrm{c}}$

$\xi_{\mathrm{p}}$

\section{Subscripts}

b

c

e

f

i

J

$\ell$

$\mathrm{m}$

n

o

$\mathrm{p}$

r

$\mathrm{s}$

w
Fraction of invaded cells for a given phase

Element of the phase mobility index takes only a binary number

Diagonal matrix with $\boldsymbol{\gamma}_{\mathbf{p}}$ in its diagonal

Phase mobility index array takes only a binary number

Phase mobility $\left(\mathrm{Pa}^{-1} \cdot \mathrm{s}^{-1}\right)$

Single-phase mobility $\left(\mathrm{Pa}^{-1} \cdot \mathrm{s}^{-1}\right)$

Mean of permeability distribution in normal domain $\left(\mathrm{m}^{2}\right)$

Phase viscosity (Pa.s)

Single-phase viscosity (Pa.s)

Mean of permeability distribution in logarithmic domain $\left(\mathrm{m}^{2}\right)$

Set of links, or connections, intersecting the $J$-th cross-sectional area

Rock porosity

Base porosity used in J-function calculations

Element of stationary distribution of invaded cells for a given phase

Element of the phase connectivity index takes only a binary number

Stationary distribution of invaded cells for a given phase

Initial state of invaded cells for a given phase

State of invaded cells at a new timestep

Phase connectivity index takes only a binary number

Standard deviation of permeability distribution in logarithmic domain $\left(\mathrm{m}^{2}\right)$

Oil-water interfacial tension $(\mathrm{N} / \mathrm{m})$

Oil-water contact angle (radians)

Percolation threshold

Fraction of open cells for a given phase

Bulk, or base depending on the context

Capillary

Entry

Fluid

Cell index

Index for cross-sectional area

Link between two connected nodes

Trapped cell index

Last-cell index, equivalent to the number of cells in a simulation model

Oil

Phase

Relative

Single-phase

Water 


\section{Superscripts}

$\begin{array}{ll}\mathrm{d} & \text { Flow direction in one of the three main principal directions } \\ \mathrm{dr} & \text { Drainage } \\ \mathrm{k} & \text { Old timestep level in percolation algorithm } \\ k+1 & \text { New timestep level in percolation algorithm } \\ \text { level } & \text { Indicate the equilibrium level of variable } \\ \max & \text { Indicate the maximum value of a variable } \\ \min & \text { Indicate the minimum value of a variable } \\ \boldsymbol{T} & \text { Transpose of an array } \\ \text { trap } & \text { Include trapping }\end{array}$

\section{Introduction}

The flow of one or more fluids in porous rocks at the field scale is modelled using Darcy's law. Multiphase flow is represented by two functions of saturation: relative permeability and capillary pressure. Relative permeability is a dimensionless quantity, which indicates the average connectivity of a rock for a given phase when it partially saturates that rock (Muskat 1937). The capillary pressure, on the other hand, is the difference in pressure between two fluid phases at equilibrium (Leverett 1941). Although this definition of capillary pressure is precise at the pore scale at fluid-fluid interfaces, it becomes less accurate for large scales where the capillary pressure may be undefined once a phase loses its connectivity across a given domain. The capillary pressure will still be defined though at the pore scale at the interface of some disconnected fluid regions. For such cases, the corresponding relative permeability value for the disconnected phase is precisely zero, and only one phase is flowing inside that domain.

\subsection{Importance of Upscaling}

There are many experimental procedures used routinely in the industry to measure multiphase flow functions (Braun and Blackwell 1981; Johnson et al. 1959; Richardson et al. 1952; Osoba et al. 1951; Jones and Roszelle 1978); however, rock samples used are typically a few $\mathrm{cm}$ across, while the flow functions need to be input into reservoir simulation models with grid-block sizes of 10-100s $\mathrm{m}$ to predict flow at the km scale. In such cases, implementing an upscaling approach is required to capture the effects of sub-scale heterogeneities and the right balance of fluid forces into Darcy-scale simulations. Therefore, the main purpose of any upscaling process in porous media is to address two main factors as we increase the scale of investigation, the change in fluid forces and heterogeneity. The main fluid forces are viscous, capillary and gravitational forces. The relative importance of these forces varies depending on many parameters and strict separation between them may not be possible (Stephen et al. 2001; Hilden and Berg 2016). Nevertheless, it is usually observed that at relatively small scales capillary forces dominate the displacement process, while viscous and gravitational forces may become more important at larger scales (Odsæter et al. 2015; Kumar and Jerauld 1996; Jonoud and Jackson 2008a).

Numerous upscaling methods are available in the literature for two-phase flow (Guzman et al. 1999; Jacks et al. 1973; Kyte and Berry 1975; Stone 1991; Pickup and Sorbie 1996; Lohne et al. 2006; Virnovsky et al. 2004; King 1989; King et al. 1993; Coll et al. 2001; Wallstrom et al. 2002; Durlofsky 1997) among which flow-based methods are considered the 
most reliable. Under this category, two fundamental approaches are employed: steady-state and dynamic pseudos. These methods are similar in concept to how multiphase flow properties are determined experimentally, but here numerical experiments are conducted. They also share the same strengths and weaknesses with their experimental counterparts (Honarpour and Mahmood 1988). Dynamic pseudo-functions are derived by performing a flow simulation in some region of a larger domain using a fine mesh and finding the average properties that reproduce the behaviour using a coarser simulation grid. In general, all dynamic pseudofunctions, produced at large scales, where viscous forces become important, depend on boundary conditions and the underlying gridding properties. They are only correct for the specific grid and flow conditions used to derive them (Barker and Thibeau 1997; Barker and Dupouy 1999).

\subsection{Steady-state Upscaling}

The main idea in steady-state upscaling is to estimate the distribution of fluid saturation and then apply a sequence of single-phase simulations for each phase individually to determine the effective multiphase flow functions. The process is repeated at different average saturations. Under specific conditions of flow, the method becomes computationally very efficient since it removes the need to run dynamic simulations for the fine-grid model. This is achieved through the assumption that only one force dominates the displacement process, while the other forces can be neglected. The term capillary limit is commonly used to describe capillary-dominated displacements, and viscous limit for viscous-dominated displacements. Gravitational forces are commonly neglected, since the method is applied to much smaller scales than those at which they become important. In general, the steady-state method gives more robust, stable and physically correct effective functions that do not depend on the details of the gridding and are less sensitive to the boundary conditions. The method could be in some cases computationally cheaper than dynamic pseudo-functions depending on flow conditions (Jonoud and Jackson 2008b, a). It becomes, however, very costly when the main fluid forces are considered in determining fluid distributions (Odsæter et al. 2015; Kumar and Jerauld 1996; Virnovsky et al. 2004; Stephen and Pickup 1999; Saad et al. 1995).

It is assumed under capillary-controlled displacements that viscous forces are negligible at the scale under investigation. This assumption only holds for regions that are away from saturation fronts, or for displacements with a large-scale capillary number that approaches zero.

Most implementations of capillary-controlled steady-state upscaling in the literature do not account for large-scale trapping since they do not permit complete disconnection of phases at the Darcy scale at a finite capillary pressure (Pickup and Stephen 1998; Ekrann and Aasen 2000). A few studies, however, have looked into this problem more carefully. Yortsos et al. (1991, 1993) considered large-scale percolation but for drainage only. They did include the effect of large-scale saturation trapping, demonstrating its importance for two-dimensional, 2D, uncorrelated media. Kueper and McWhorter (1992) introduced a macroscopic invasion percolation theory to upscale capillary pressure functions. They accounted for trapped cells using a percolation algorithm, but did not consider the impact on average saturation as a consequence of trapping inside these cells. In addition, the study did not compute average relative permeabilities and only considered 2D domains.

In this study, we focus on steady-state upscaling for capillary-controlled displacements, where we allow complete disconnection of phases at the Darcy scale, accounting for largescale trapping of fluids. For this, large-scale invasion percolation is used as outlined by Kueper and McWhorter (1992) and Yortsos et al. (1991, 1993), but extended to waterflooding, 
exploring the effects of wettability, while unambiguously accounting for large-scale fluid trapping inside trapped cells. This is illustrated using random permeability fields in 2D and 3D domains, correlated with local capillary pressure-saturation relationships using Leverett J-function scaling, assuming that heterogeneity in capillary pressure is connected primarily to heterogeneity in permeability and porosity. We also use characteristic J-function and local relative permeability curves that are typical for water-wet, oil-wet and mixed-wet systems.

We refer in this paper to the wetting phase as water and the non-wetting phase as oil, although the process is applicable to any pairs of immiscible fluids, including $\mathrm{CO}_{2}$-brine systems where large-scale trapping is extremely important for storage security (Saadatpoor et al. 2010; Juanes et al. 2006).

\section{Darcy-scale Saturation-Dependent Functions}

Before going into the detail of large-scale trapping mechanisms, we define unambiguously large-scale saturation-dependent functions used in Darcy-scale simulations. Figure 1 shows typical capillary pressures and relative permeabilities for drainage and waterflooding. The main point here is that we do not allow the capillary pressure to diverge to infinity at end-point saturations: irreducible water $S_{\text {wir }}$ and residual oil saturations $S_{\text {orw }}$. Instead, the functions are bounded by a maximum and a minimum value, $p_{\mathrm{c}}^{\max }$ and $p_{\mathrm{c}}^{\min }$. To identify these points, the corresponding relative permeability functions are used, for when they are zero at the end-point saturations, the limits on the capillary pressure functions are well-defined. In this case, the phases are completely disconnected at the pore level, forming isolated clusters that are completely immobile.

In other cases, $p_{\mathrm{c}}^{\max }$ and $p_{\mathrm{c}}^{\min }$ may correspond to relative permeability values that are not zero but extremely small to the point that they can be considered immobile. Upon a closer look at the pore level, such a phase might be connected in thin layers (Valvatne and Blunt 2004). At larger scales, this layer flow might be neglected considering the timescale of the process (Blunt 2017). Here, a threshold value $k_{\mathrm{rf}}^{\mathrm{c}}$ is imposed, below which layer flow is ignored. Having said this, there are no clear guidelines on how to find this cut-off value; it is case dependent. This will be investigated more thoroughly in future studies. In this study, we use a well-defined limits on capillary pressure functions, corresponding to zero relative permeabilities at end-point saturations.

If instead we only allowed the relative permeability to be zero at an infinite capillary pressure, then we would never disconnect phases in a steady-state simulation, since-for any finite imposed capillary pressure - the flow of both phases would be finite. We would then be unable to capture large-scale trapping; the residual saturation, regardless of heterogeneity, would be the residual saturation in the input relative permeability.

\section{Theory of Large-scale Trapping of Fluids}

There are two main trapping mechanisms that must be accounted for when calculating average fluid volumes and connectivities. These occur at the large scale and are distinct from the local trapping at the pore scale that is accounted for by end-point saturations in the local capillary pressures and relative permeabilities, mentioned above. The first mechanism is the trapping of water by oil during drainage. As oil invades the system at a given position of capillary equilibrium, referred to as $p_{\mathrm{c}}^{\text {level }}$, some regions of the domain may trap some movable water 


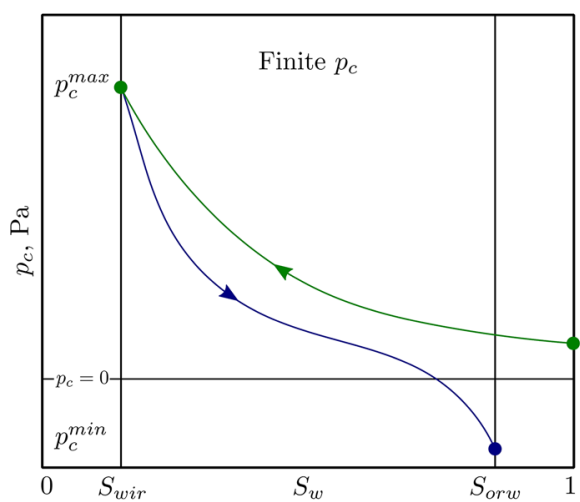

(a)

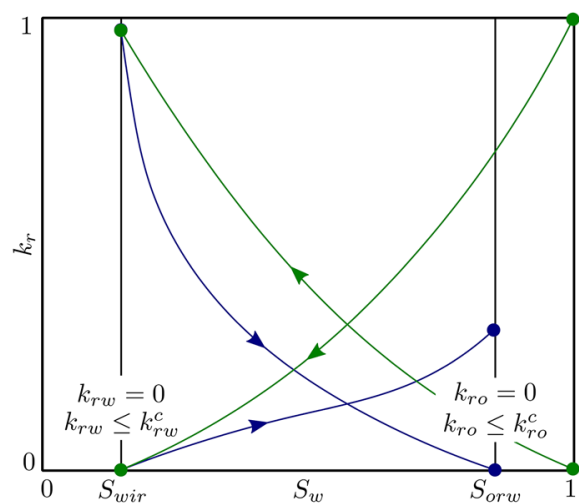

(b)

Fig. 1 A schematic of saturation-dependent functions defined at large scales. a Typical capillary pressures for drainage (green) and waterflooding (blue). b Typical relative permeabilities for drainage (green) and waterflooding (blue). Capillary pressures are bounded by $p_{\mathrm{c}}^{\max }$ and $p_{\mathrm{c}}^{\min }$, corresponding to $k_{\mathrm{rf}}=0$, or $k_{\mathrm{rf}} \leq k_{\mathrm{rf}}^{\mathrm{c}}$, for $f=\{\mathrm{o}, \mathrm{w}\}$

as movable water as movable oil fills completely neighbouring regions in which the relative permeability to water $k_{\mathrm{rw}}$ drops to zero. The trapped water volumes in these regions cannot be estimated from the local capillary pressure-saturation relationship $p_{\mathrm{c}}\left(S_{\mathrm{w}}\right)$ at a given $p_{\mathrm{c}}^{\text {level }}$, as in conventional approaches, but rather a special treatment is required. Figure 2 demonstrates this trapping mechanism graphically in 2D systems, where the relevant cells are labelled from 1 to 5. Corresponding drainage capillary pressures are also shown in Fig. 2b. As fluids are brought to equilibrium at discrete, but elevated levels, water is trapped, locally, once $p_{\mathrm{c}}^{\text {level }}$ reaches $p_{\mathrm{c}}^{\max }$ in a given cell. From the local capillary pressures in Fig. $2 \mathrm{~b}$, this local trapping of water takes place in the following order: cells 1,2,3 and 4. Large-scale trapping only happens when $p_{\mathrm{c}}^{\text {level }}$ reaches $p_{\mathrm{c}}^{\max }$ of cell 4 , at which the movable water in cell 5 becomes disconnected from the rest of the water in the system. As $p_{\mathrm{c}}^{\text {level }}$ increases above $p_{\mathrm{c}}^{\text {max }}$ of cell $4, S_{\mathrm{W}}$ in cell 5 will remain fixed at $S_{\mathrm{w}}^{\text {trap }}$, as indicated in Fig. $2 \mathrm{~b}$, and will not follow the local capillary pressure defined for that cell.

The second trapping mechanism is illustrated in Fig. 3. Here, oil is trapped by onlywater-movable regions. The process occurs during waterflooding, in mixed-wet and oil-wet systems. As $p_{\mathrm{c}}^{\text {level }}$ decreases, the water saturation increases until, in some regions, the relative permeability to oil $k_{\text {ro }}$ drops to zero, trapping movable oil in surrounded regions. This is shown in Fig. $3 \mathrm{~b}$ where as $p_{\mathrm{c}}^{\text {level }}$ drops below $p_{\mathrm{c}}^{\min }$ of cell 4 , oil is trapped in cell 5 and stays fixed at $S_{\mathrm{W}}^{\text {trap }}$ as $p_{\mathrm{c}}^{\text {level }}$ continues to decline. The water saturation does not change in cell 5, since there cannot be any displacement of the trapped oil. In Fig. 3a, the arrangement of cells is shown in the 2D system, where blue indicates cells with local trapping of oil, while red indicates cells with large-scale trapping.

\section{Steady-State Upscaling: Revised Procedure}

In this section, we give a detailed description of the revised two-phase capillary-controlled steady-sate upscaling method. In the capillary limit, it is assumed that fluids are distributed inside a region of interest in such a way that minimizes capillary pressure gradients. In 


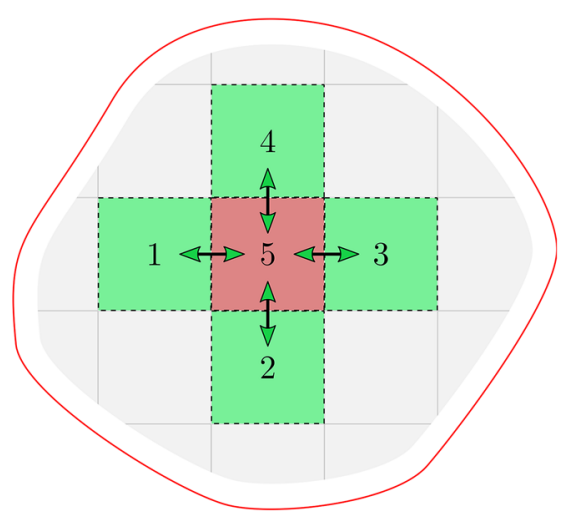

(a)

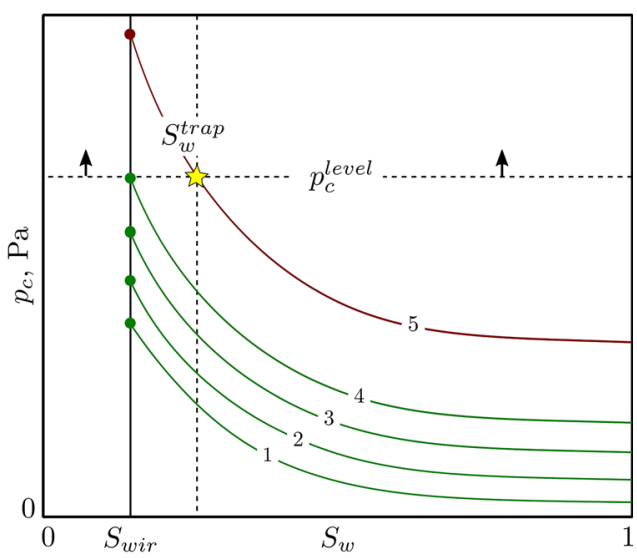

(b)

Fig. 2 An illustration of capillary trapping during drainage at the Darcy scale in 2D systems. a The arrangement of cells in the 2D system, showing their labels. Green indicates cells with local-scale trapping of water, while red indicates cells with large-scale trapping. b Corresponding local capillary pressure functions demonstrating the trapped water saturation $S_{\mathrm{W}}^{\text {trap }}$ in cell 5

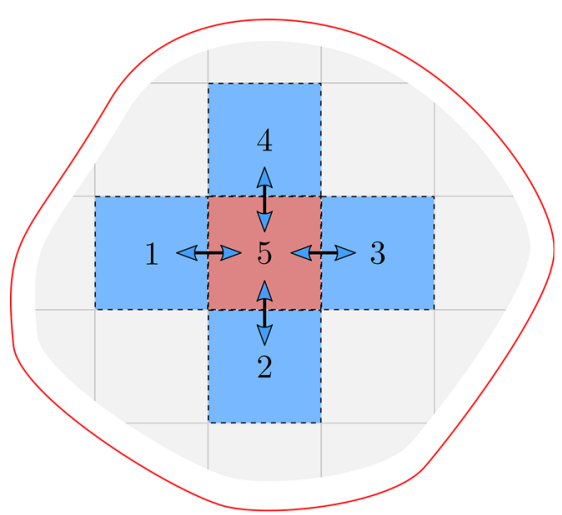

(a)

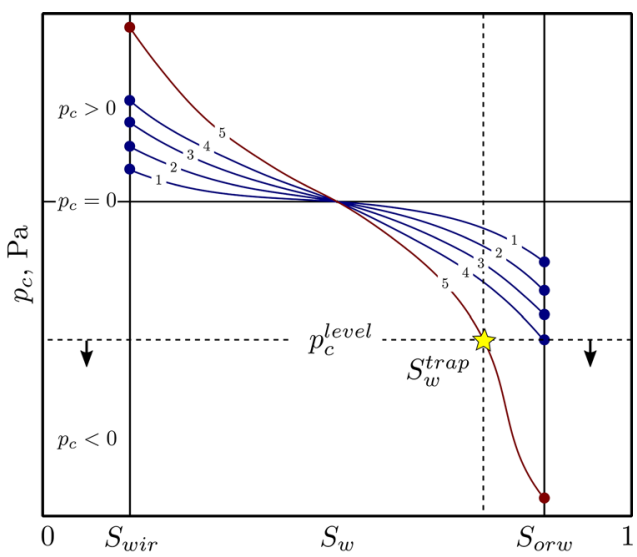

(b)

Fig. 3 An illustration of capillary trapping during waterflooding at the Darcy scale in 2D systems. a The arrangement of cells in the 2D system, showing their labels. Blue indicates cells with local-scale trapping of oil, while red indicates cells with large-scale trapping. b Corresponding local capillary pressure functions demonstrating the trapped water saturation $S_{\mathrm{W}}^{\text {trap }}$ in cell 5

the complete absence of other forces, capillary equilibrium is achieved when the gradient of capillary pressure approaches zero, resulting in a stationary distribution of fluids that is unique for the given set of initial and boundary conditions. At the Darcy scale, capillary equilibrium can be achieved by running a dynamic simulation for a given set of initial and boundary conditions until reaching a stationary fluid distribution. This is, however, a computationally very expensive procedure. A much more efficient and simpler way is to estimate the fluid distribution without running a dynamic simulation. 
Fig. 4 Computations of $S_{\mathrm{W}}$ from waterflood $p_{\mathrm{c}}\left(S_{\mathrm{W}}\right)$ at three values of $p_{\mathrm{c}}^{\text {level }}$ in a system with three local capillary pressures labelled $i, j$ and $k$. When $p_{\mathrm{c}}^{\text {level }}=6 \mathrm{kPa}$, cell $j$ does not allow water to move across it as the relative permeability to water is zero; only oil is mobile. At $p_{\mathrm{c}}^{\text {level }}=0$ $\mathrm{kPa}$, both phases are mobile through all cells, whereas when $p_{\mathrm{c}}^{\text {level }}=-6 \mathrm{kPa}$, cell $j$ becomes disconnected to oil as only water is allowed to move across it. In this last case, the relative permeability to oil is zero

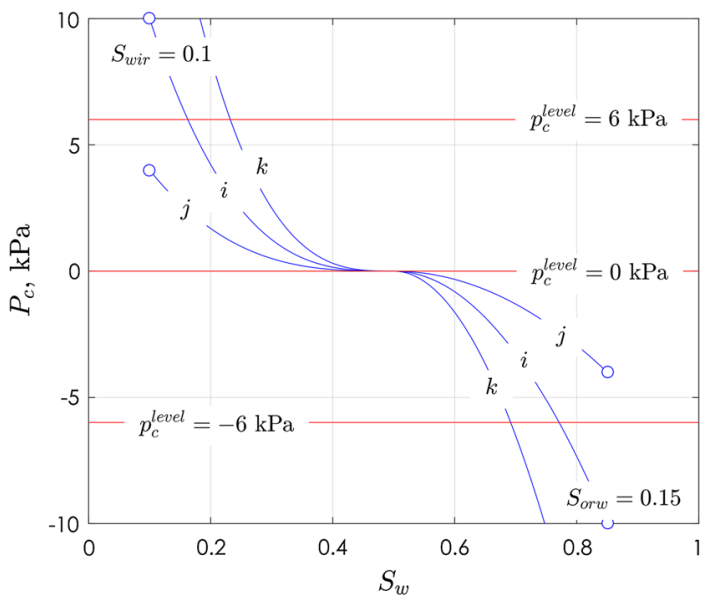

The key idea is to use the local capillary pressure-saturation relationships that are already known at the grid-block scale and use them to estimate directly the saturation distributions. The main problem with this approach, which can be avoided by using dynamic simulations, is that fluid volumes required to achieve a certain capillary equilibrium state at a given local region might not be reached since there is no connected path for that fluid to flow to that region. In other words, a phase must make a connected path from inlet boundaries to the region of interest. From local relative permeabilitysaturation relationships, it is possible to identify these regions that do not allow a given phase to flow through them. This concept is illustrated graphically in Fig. 4, in which the water saturation distribution is estimated at three positions of $p_{\mathrm{c}}^{\text {level }}$. The presented capillary pressures are typical for a waterflooding process in a mixed-wet system. When $p_{\mathrm{c}}^{\text {level }}=6 \mathrm{kPa}$, cell $j$ does not allow water to move across it as the relative permeability to water is zero; only oil is mobile. At $p_{\mathrm{c}}^{\text {level }}=0 \mathrm{kPa}$, both phases are mobile through all cells, whereas when $p_{\mathrm{c}}^{\text {level }}=-6 \mathrm{kPa}$, cell $j$ becomes disconnected to oil as only water is allowed to move across it. In this last case, the relative permeability to oil is zero.

The revised upscaling process then starts by assigning a capillary pressure level, $p_{\mathrm{c}}^{\text {level}}$, at which capillary equilibrium is imposed for the connected phases in the domain. From local capillary pressure curves, the saturation distribution is estimated. The corresponding relative permeability values are also computed from local relative permeability curves, $k_{\mathrm{rp}}\left(S_{\mathrm{w}}\right)$ for $p=\{\mathrm{o}, \mathrm{w}\}$, and used to indicate whether a cell, or a node, can percolate a given phase across it or not. Once local non-percolating cells are identified, a solver that fills cells and determines whether a phase forms a connected cluster that spans the whole domain, is called; we refer to them as invaded cells. Such a condition confirms the presence of a global connectivity of the system. The solver is also used to identify cells that can percolate a given phase but could not be reached as a result of being locked in by neighbouring non-percolating cells. These are termed trapped cells, and they do not contribute to the overall connectivity of the system. In addition, special treatment is required to account for the trapping of fluids inside these trapped cells, as described previously in Sect. 3. Algorithms developed to tackle such problems are described next. 


\section{Filling Cells at a Given Capillary Pressure}

In this section, we describe the algorithm used to determine invaded cells in a system. We simply describe how to invade cells for a given, specified capillary pressure. In the following section (Sect. 6), we describe how we account for trapping of fluids as the imposed capillary pressure is varied during a displacement. We here start by defining two main index functions, the phase mobility and the phase connectivity indices as detailed below, where subsequent calculations depend on both of them. We introduce a simple and efficient algorithm that works for structured and unstructured simulation grids.

\subsection{Phase Mobility Index $\gamma_{p}$}

The mobility index, $\boldsymbol{\gamma}_{\mathbf{p}}$, for a given phase, $\mathrm{p}$, is an array defined as:

$$
\gamma_{\mathbf{p}}=\left[\gamma_{\mathrm{p}_{1}}, \gamma_{\mathrm{p}_{2}}, \ldots, \gamma_{\mathrm{p}_{n}}\right]^{T},
$$

where $\mathrm{p}$ refers to either oil, denoted as o, or water, denoted as $\mathrm{w}$. The array has $n$ elements equivalent to the number of cells in a simulation grid. The elements of $\gamma_{\mathbf{p}}$ are defined as follows:

$$
\gamma_{\mathrm{w}_{i}}= \begin{cases}0, & p_{\mathrm{c}_{i}}^{\max } \leq p_{\mathrm{c}}^{\text {level }} \\ 1, & \text { otherwise }\end{cases}
$$

and,

$$
\gamma_{\mathrm{o}_{i}}= \begin{cases}0, & p_{\mathrm{c}_{i}}^{\min } \geq p_{\mathrm{c}}^{\text {level }}, \\ 1, & \text { otherwise. }\end{cases}
$$

Equations 2 and 3 state that a cell $i$ takes a value of 0 if a phase is immobile, otherwise the cell takes a value of 1 . If the oil phase, for instance, is immobile for a given cell $i$, then $\gamma_{o_{i}}=0$ for that cell, otherwise $\gamma_{\mathrm{o}_{i}}=1$. The index $\gamma_{\mathbf{p}}$, therefore, is used to identify cells that have the ability to transmit a given phase and hence is termed the phase mobility index.

The process here represents a single step in a classic invasion percolation algorithm where $\gamma_{\mathbf{p}}$ indicates whether a phase can potentially flow across a given cell at a specified $p_{\mathrm{c}}^{\text {level }}$ or not (Kueper and McWhorter 1992; Yortsos et al. 1991, 1993; Hunt et al. 2014). The next step then is to use this mobility index to determine whether a given phase forms a connected path across a system or not. This is described below.

\subsection{Phase Connectivity Index $\psi_{\mathrm{p}}$}

The phase connectivity index, $\psi_{\mathbf{p}}$, for a given phase, $\mathrm{p}$, is a vector defined as:

$$
\psi_{\mathbf{p}}=\left[\psi_{\mathrm{p}_{1}}, \psi_{\mathrm{p}_{2}}, \ldots, \psi_{\mathrm{p}_{n}}\right]^{T}, \quad p \in\{\mathrm{o}, \mathrm{w}\},
$$

where the subscript $\mathrm{p}$ refers to either oil, denoted as o, or water, denoted as $\mathrm{w}$. The vector has $n$ elements equivalent to the number of cells in the simulation model. The elements of $\psi_{\mathbf{p}}$ are either 0 or 1 , where 1 indicates that a cell is invaded, otherwise it is not. The index function $\psi_{\mathbf{p}}$, therefore, is used to indicate cells that are connected to the main cluster of a phase. The evolution of $\psi_{\mathbf{p}}$ with time is governed by

$$
\psi_{\mathbf{p}}^{k+1}=A_{\mathbf{p}} \psi_{\mathbf{p}}^{k}, \quad p \in\{\mathrm{o}, \mathrm{w}\},
$$

where the superscript k refers to the timestep number and $\boldsymbol{A}_{\mathbf{p}}$ is a symmetric $n$-by- $n$ matrix, given by

$$
\boldsymbol{A}_{\mathbf{p}}=\boldsymbol{\Gamma}_{\mathbf{p}}(\boldsymbol{B}+\boldsymbol{I}) \boldsymbol{\Gamma}_{\mathbf{p}}, \quad p \in\{\mathrm{o}, \mathrm{w}\},
$$


where $\boldsymbol{\Gamma}_{\mathbf{p}}$ is an $n$-by- $n$ diagonal matrix having the vector $\boldsymbol{\gamma}_{\mathbf{p}}$ in its diagonal, $\boldsymbol{B}$ is the adjacency matrix and $\boldsymbol{I}$ is the identity matrix. The adjacency matrix $\boldsymbol{B}$ is a square matrix in which the number of rows and columns is equal to the number of cells in a simulation model. The elements of this matrix are either 0 or 1 , where 1 indicates that there is a connection, or a link, between a pair of cells (West 2001). More formally, the matrix can be constructed for any simulation model as follows:

$$
B_{i j}= \begin{cases}1, & \text { if cell } i \text { is connected to cell } j, \\ 0, & \text { otherwise, }\end{cases}
$$

where $B_{i j}$ refers to the element in row $i$ and column $j$ of the matrix $\boldsymbol{B}$.

The matrix $\boldsymbol{\Gamma}_{\mathbf{p}}$ has the following structure:

$$
\boldsymbol{\Gamma}_{\mathbf{p}}=\left(\begin{array}{cccc}
\gamma_{\mathrm{p}_{1}} & & & \\
& \gamma_{\mathrm{p}_{2}} & & \\
& & \ddots & \\
& & & \gamma_{\mathrm{p}_{n}}
\end{array}\right), \quad p \in\{\mathrm{o}, \mathrm{w}\} .
$$

Equation 5 is a first-order difference equation that describes the evolution of invaded cells through a model. The process usually starts from one of the boundaries by providing an initial state of invaded cells, $\psi_{\mathbf{p}}^{0}$, where, as previously mentioned, the integer 1 indicates that a cell is invaded, while 0 indicates it is not. The invaded cells are then updated using Eq. 5. Because of the way the matrix $\boldsymbol{A}_{\mathbf{p}}$ is defined in Eq. 6, at each iteration, $\boldsymbol{\psi}_{\mathbf{p}}^{k+1}$ stores positive integer numbers which might be larger than one, indicating the number of possible visits to a given cell. For the purpose of our algorithm, all nonzero elements are reset to 1 at the end of each update. Iterations are then performed until the number of invaded cells does not change over time, at which the loop is terminated. This stationary distribution of invaded cells is referred to as $\psi_{\mathbf{p}}^{\infty}$ and is used in all subsequent calculations. In practice, the state of the cells at the far boundary from where the process has started are checked. If at least one of the cells has been invaded, $\psi_{\mathrm{p}_{\mathrm{i}}}^{\infty}=1$, then a given phase is said to have established a global connectivity across the system at the specified $p_{\mathrm{c}}^{\text {level }}$. Calculations can then be performed to determine average relative permeabilities, where only invaded cells are taken into consideration. The overall algorithm for this filling process is shown in Fig. 5.

From the elements of $\gamma_{\mathbf{p}}$ and $\boldsymbol{\psi}_{\mathbf{p}}^{\infty}$, we calculate two important average quantities of the system. The first one is $\xi_{\mathrm{p}}$, which describes the fraction of open cells, or cells that are accessible to a given phase. This parameter is particularly important in basic percolation theory, from which a critical fraction $\xi_{\mathrm{c}}$ is identified and used to indicate precisely when a global connectivity develops; it is universal for randomly disordered and infinitely large systems (Hunt et al. 2014; Grimmett 1999). $\xi_{\text {p }}$ can be calculated as

$$
\xi_{\mathrm{p}}=\frac{1}{N} \sum_{i=1}^{n} \gamma_{\mathrm{p}_{i}}, \quad p \in\{\mathrm{o}, \mathrm{w}\},
$$

where $N$ is the total number of cells and $\gamma_{\mathrm{p}_{i}}$ is the phase mobility index defined previously in Eqs. 1, 2 and 3.

The second quantity is $\eta_{\mathrm{p}}$, which is defined as the fraction of invaded cells; it is given by

$$
\eta_{\mathrm{p}}=\frac{1}{N} \sum_{i=1}^{n} \psi_{\mathrm{p}_{i}}^{\infty}, \quad p \in\{\mathrm{o}, \mathrm{w}\},
$$




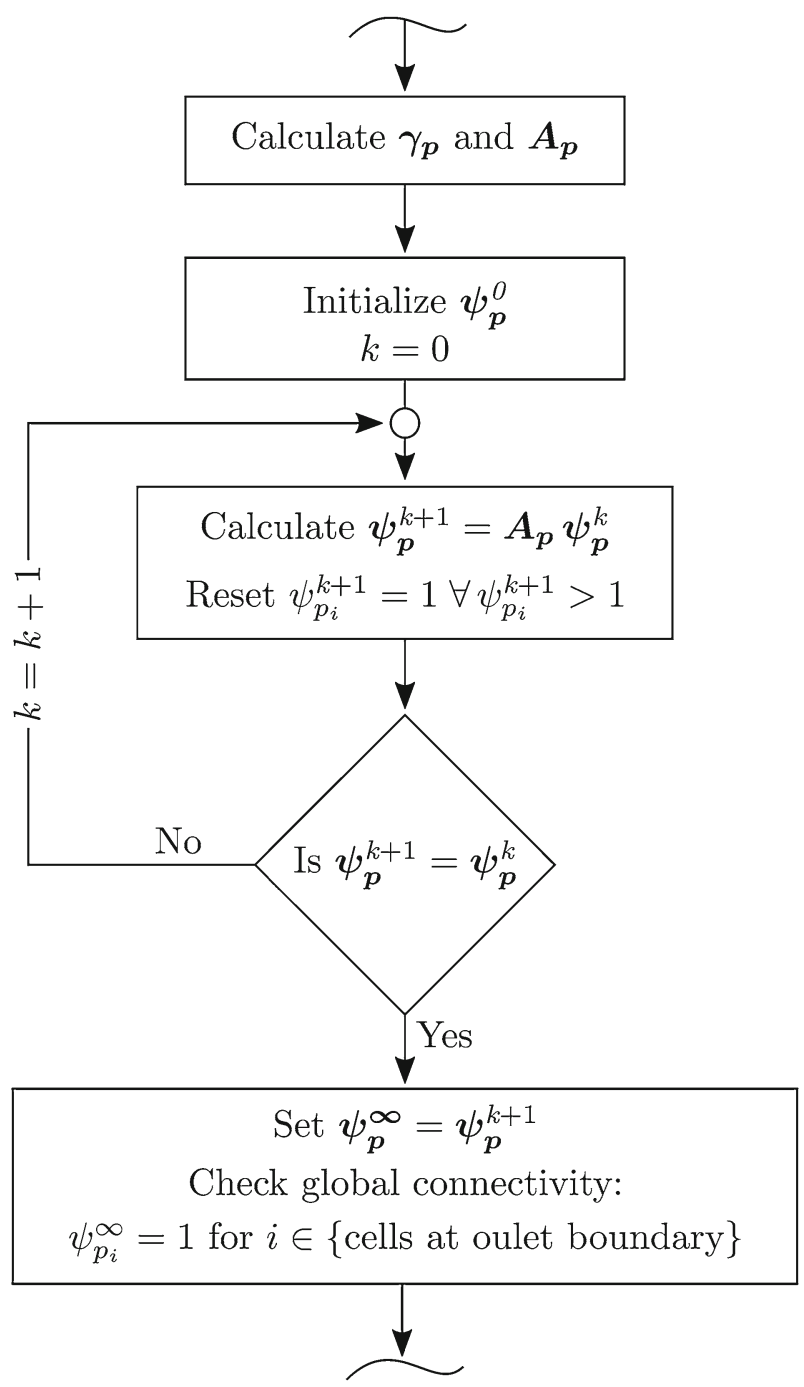

Fig. 5 A flowchart showing the algorithm for filling cells at a fixed $p_{\mathrm{c}}^{\text {level }}$ for both oil and water

where $\psi_{\mathrm{p}_{i}}^{\infty}$ is the elements of the stationary distribution of invaded cells defined previously in Sect 5.2. These parameters will be used in Sect. 8 to validate the percolation solver for 2D and $3 \mathrm{D}$ systems.

\section{Large-scale Invasion Percolation with Trapping}

We now describe our large-scale invasion percolation algorithm that accounts for largescale saturation trapping. In the previous section, we showed how to fill cells-finding invaded cells-at a given $p_{\mathrm{c}}^{\text {level }}$, but here we allow $p_{\mathrm{c}}^{\text {level }}$ to vary between different equi- 
librium positions. We also show how we determine the saturation distribution at each $p_{\mathrm{c}}^{\text {level }}$, while accounting for fluid trapping.

A flowchart that illustrates this is shown in Fig. 6. We start first by determining the $\boldsymbol{p}_{\mathbf{c}}^{\text {level }}$ distribution in which the events of trapping are represented by its elements, $p_{\mathrm{c}_{j}}^{\text {level }}$. This distribution can be estimated either from $\boldsymbol{p}_{\mathbf{c}}^{\max }$ for drainage, or from $\boldsymbol{p}_{\mathbf{c}}^{\min }$ for waterflooding, corresponding for maximum and minimum capillary pressure distributions, respectively. This will be explained more in Sect. 9 when we show our examples.

Before iterating on $p_{\mathrm{c}_{j}}^{\text {level }}, \boldsymbol{p}_{\mathbf{c}}^{\text {level }}$ is sorted either in descending or in ascending order, depending on the displacement process being modelled. In waterflooding, for instance, the distribution is sorted in descending order, resembling the process for steady-state experiments, in which capillary pressure is brought to equilibrium in discrete levels from a higher to a lower equilibrium state. On the other hand, the distribution for drainage is sorted in ascending order as capillary equilibrium rises incrementally. Then, we initialize an array, $\boldsymbol{p}_{\mathbf{c}}^{\text {trap }}$, to store the capillary pressure levels, or events, at which trapping happens in each cell. The length of this array is equivalent to the number of cells $N$ in the model. $p_{\mathbf{c}}^{\text {trap }}$ is initialized to zero, indicating that all cells are initially not trapped.

We then start the iterations at a given $p_{\mathrm{c}_{j}}^{\text {level }}$, from which the mobility indices, $\boldsymbol{\gamma}_{\mathbf{w}}$ and $\gamma_{\mathbf{0}}$, are computed as in Eqs. 2 and 3. After that, the solver described in Fig. 5 is called to determine the stationary distribution of invaded cells, $\psi_{\mathbf{p}}^{\infty}$. At the current position of $p_{\mathbf{c}_{j}}^{\text {level }}$, trapped cells $m$ are identified as those that allow a phase to move across them $\left(\gamma_{\mathrm{p}_{\mathrm{m}}}=1\right)$, but have not been invaded at the current event $\left(\psi_{\mathrm{pm}}^{\infty}=0\right)$, giving that they have not been trapped from previous events $\left(p_{\mathrm{c}_{\mathrm{m}}}^{\text {trap }}=0\right)$. This is a crucial step in the process since we need to find the new trapped cells at the large scale and at the current $p_{\mathrm{c}_{j}}^{\text {level }}$. These conditions represent the trapping mechanisms as detailed in Sect. 3. We also exclude cells with $\left(\gamma_{\mathrm{p}_{\mathrm{m}}}=0\right)$ as they represent cells with local trapping.

The trapped cells $\mathrm{m}$ are assigned then to the current $p_{\mathrm{c}_{j}}^{\text {level }}$ where this value will stay constant. This condition is stated in Fig. 6 in set-builder notation. The average water saturation $\tilde{S}_{\mathrm{w}}$ can then be calculated at the end of each iteration, as follows

$$
\tilde{S}_{\mathrm{W}}=\frac{\sum_{i=1}^{n} \phi_{i} V_{\mathrm{b}_{i}} S_{\mathrm{w}_{i}}}{\sum_{i=1}^{n} \phi_{i} V_{\mathrm{b}_{i}}},
$$

where $\phi_{i}$ is the porosity, $V_{\mathrm{b}_{i}}$ is the bulk volume in $\mathrm{m}^{3}$, and $S_{\mathrm{w}_{i}}$ is the water saturation of cell $i$ determined inversely from local $p_{\mathrm{c}_{i}}\left(S_{\mathrm{W}}\right)$, where $p_{\mathrm{c}_{j}}^{\text {level }}$ is used for non-trapped cells $\left(p_{\mathrm{c}_{i}}^{\text {trap }}=0\right)$, and nonzero elements of $p_{\mathbf{c}}^{\text {trap }}$ are used for trapped cells.

\section{Average Relative Permeability Algorithm}

The computation of average relative permeability is described by a flowchart in Fig. 7. At a given capillary equilibrium state, $\xi_{\mathrm{p}}$ is computed and checked. When $\xi_{\mathrm{p}} \neq 1$, this indicates that some cells with local trapping are present which might produce some large-scale trapping regions. In this case, a percolation solver is called to confirm global connectivity of a phase. Once confirmed, a flow solver is called to calculate $\tilde{k}_{\mathrm{rp}}^{\mathrm{d}}$ using Eq. 12. The key step here is to remove the trapped cells at both the local and the large scales from the main matrix, since 


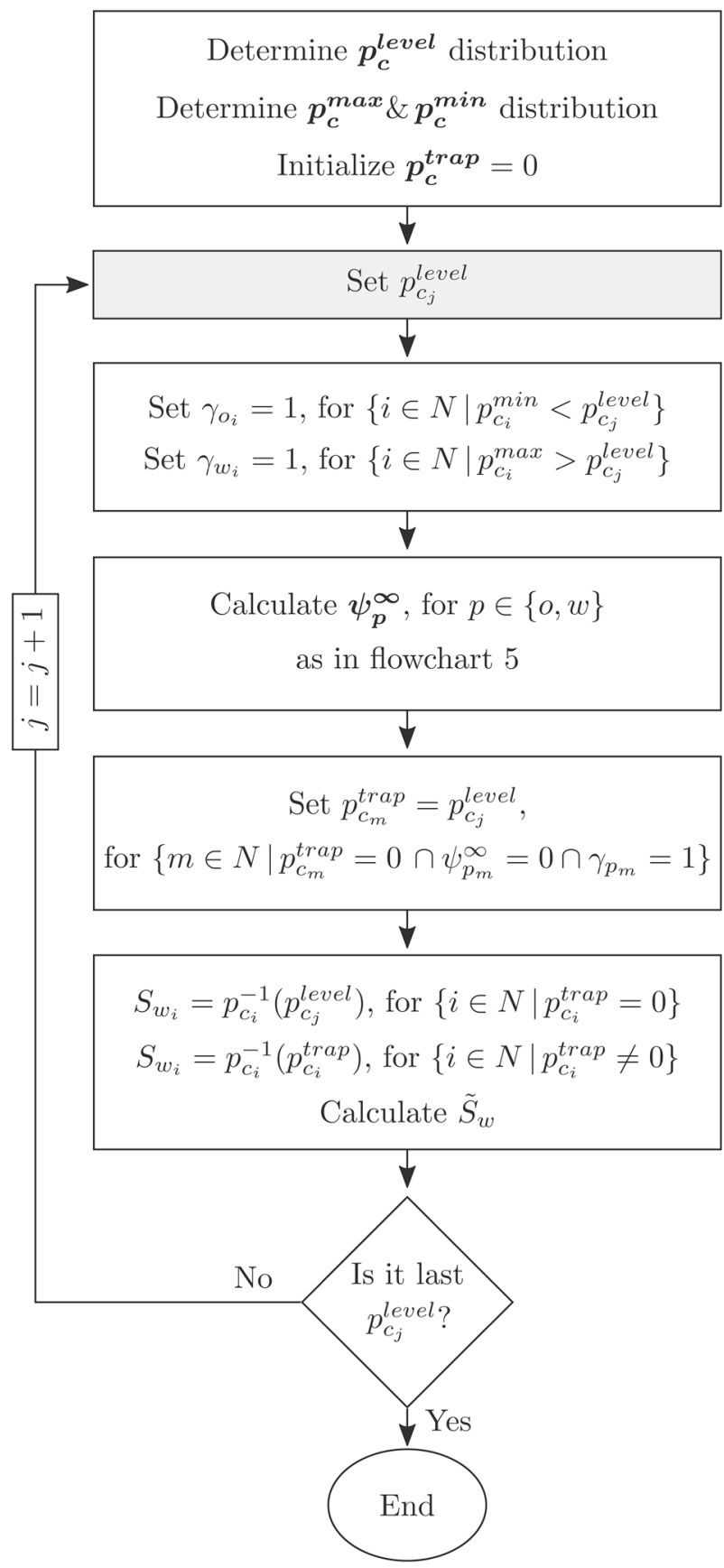

Fig. 6 A flowchart illustrating the algorithm for macroscopic invasion percolation where $p_{\mathrm{c}}^{\text {level }}$ is varied. This also shows the computations of average fluid saturation at each $p_{\mathrm{c}}^{\text {level }}$ accounting for fluid trapping for both oil and water 


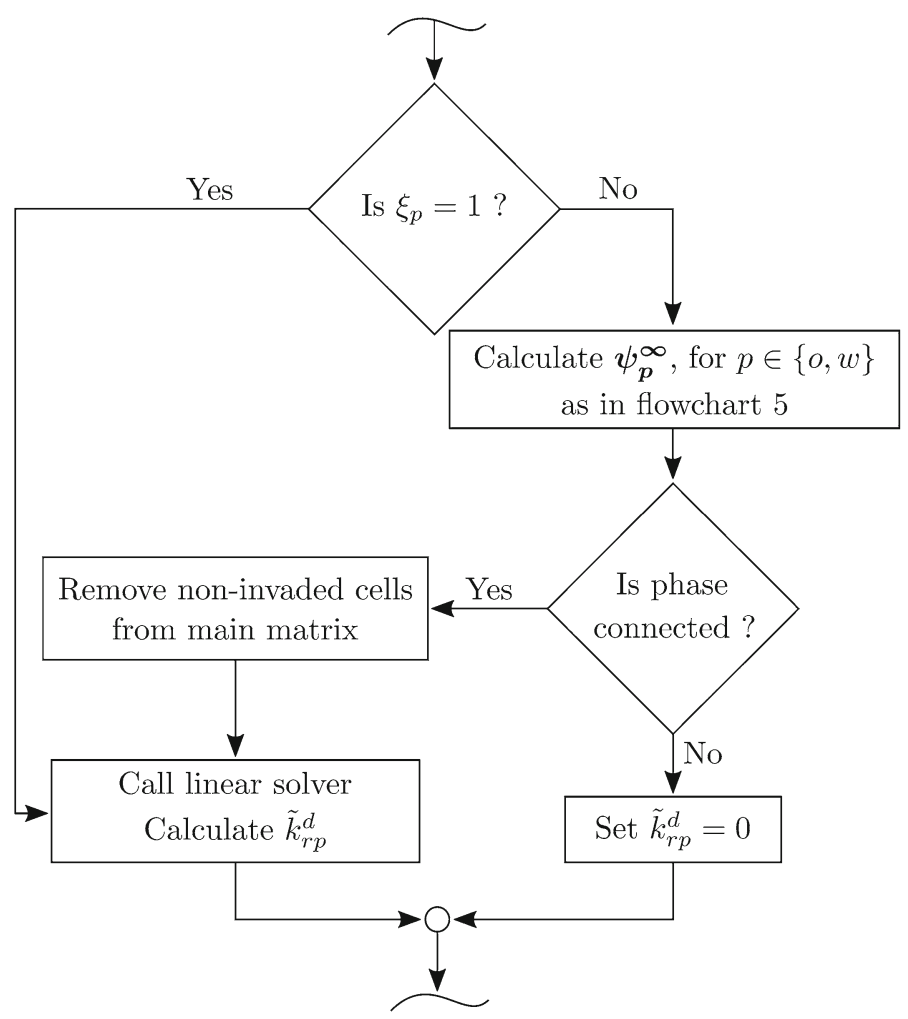

Fig. 7 A flowchart demonstrating the computations of average relative permeabilities for oil and water, considering the impact of invasion percolation with trapping

they do not contribute to the flow, and their inclusion could make the matrix ill-conditioned or even singular.

The average relative permeabilities are computed from:

$$
\tilde{k}_{\mathrm{rp}}^{\mathrm{d}}=\frac{\mu_{\mathrm{p}}}{\mu_{\mathrm{s}}} \frac{\tilde{Q}_{\mathrm{p}}^{\mathrm{d}}}{\tilde{Q}_{\mathrm{s}}^{\mathrm{d}}}, \quad p \in\{\mathrm{o}, \mathrm{w}\}, \quad d \in\{x, y, z\},
$$

where $\tilde{k}_{\mathrm{rp}}^{\mathrm{d}}$ is defined as the average relative permeability for a phase $\mathrm{p}$ in the direction d parallel to one of the coordinate axes of the three-dimensional system, $\mu_{\mathrm{p}}$ is the phase viscosity in Pa.s and $\mu_{\mathrm{s}}$ is the fluid viscosity used in single-phase calculations. The parameter $\tilde{Q}^{\mathrm{d}}$ is the average volumetric flux within a volume of interest in a direction $\mathrm{d}$ in $\mathrm{m}^{3} / \mathrm{s}$. The subscripts $\mathrm{p}$ and $\mathrm{s}$ are used to indicate whether the volumetric flux is obtained from two-phase or singlephase flow calculations, respectively. Both functions, $\tilde{Q}_{\mathrm{p}, \mathrm{s}}^{\mathrm{d}}$, can be computed from numerical simulations as follows:

$$
\tilde{Q}_{\mathrm{p}, \mathrm{s}}^{\mathrm{d}}=\frac{1}{m^{\mathrm{d}}} \sum_{J=1}^{m^{\mathrm{d}}} \sum_{\ell \in \Omega_{J}} Q_{\{\mathrm{p}, \mathrm{s}\}_{\ell}}, \quad p \in\{\mathrm{o}, \mathrm{w}\}, \quad d \in\{x, y, z\},
$$

where $m^{\mathrm{d}}$ is the total number of cross-sectional areas in the averaged volume perpendicular to a flow in direction $\mathrm{d}, Q_{\mathrm{p} \ell}$ is a volumetric flux across a link $\ell$ between two neighbouring 
nodes in $\mathrm{m}^{3} / \mathrm{s}$ and $\Omega_{J}$ here indicates the set of links, or connections, intersecting the $J$-th cross-sectional area. The way to use Eq. 13 to compute average flow rates is, therefore, to first calculate total flow rates across each cross-sectional area and then to average them out. If we allow, for instance, a flow along the $\mathrm{x}$-axis in a 3D system with a cubic grid, we first sum the fluxes from the cells intersecting a single plane in the $\mathrm{y}-\mathrm{z}$ direction. We record this value and repeat the process in all other parallel planes. The number of planes in this case is equivalent to $m^{\mathrm{d}}$. We then average these fluxes as shown in Eq. 13.

Similarly for $\tilde{Q}_{\mathrm{s}}^{\mathrm{d}}$, average volumetric flux for single-phase flow, the calculations are performed as with $\tilde{Q}_{\mathrm{p}}^{\mathrm{d}}$ in Eq. 13. The only difference is in the definition of the volumetric flux $Q_{\mathrm{p}, \mathrm{s}}$ across any pair of nodes $i$ and $j$. This is given by the multiphase Darcy equation:

$$
Q_{\{\mathrm{p}, \mathrm{s}\}_{i j}}=\frac{k_{i j} A_{i j}}{L_{i j}} \lambda_{\{\mathrm{p}, \mathrm{s}\}_{i j}}\left(P_{\{\mathrm{p}, \mathrm{s}\}_{i}}-P_{\{\mathrm{p}, \mathrm{s}\}_{j}}\right), \quad p \in\{\mathrm{o}, \mathrm{w}\},
$$

where $k_{i j}$ is the absolute permeability of the link in $\mathrm{m}^{2}$, which is calculated as the harmonic mean of the absolute permeability of the nodes $i$ and $j:\left(\frac{2 k_{i} k_{j}}{k_{i}+k_{j}}\right), A_{i j}$ is the cross-sectional area between the two nodes in $\mathrm{m}^{2}, L_{i j}$ is the length between them in $\mathrm{m}$, and $\lambda_{\mathrm{p}_{i j}}$ is the phase mobility defined as, for two-phase flow $\lambda_{\mathrm{p}_{i j}}=\frac{k_{\mathrm{rp}}\left(S_{\mathrm{w}_{i j}}\right)}{\mu_{\mathrm{p}}}$, while for single-phase flow it is given by $\lambda_{\mathrm{s}_{i}}=\mu_{\mathrm{s}}^{-1}$. The phase mobility in two-phase flow is calculated from individual nodes as $\lambda_{\mathrm{p}_{i j}}=\frac{1}{2}\left(\lambda_{\mathrm{p}_{i}}+\lambda_{\mathrm{p}_{j}}\right)$, given that the saturation distribution is already known as previously described in Sect. 4. In Eq. 14, $P_{\{\mathrm{p}, \mathrm{s}\}}$ is the phase pressure in Pa for two-phase $P_{\mathrm{p}}$ or single-phase $P_{\mathrm{s}}$ flow calculations. They are determined by solving a system of equations constructed by applying material-balance principles, from which we obtain: $\sum_{j} Q_{\{\mathrm{p}, \mathrm{s}\}_{i j}}=0$.

For the calculation of average relative permeabilities, it is important to apply the same set of boundary conditions for pressure when solving for $P_{\mathrm{p}}$ and $P_{\mathrm{s}}$. In our solution, a pressure boundary condition is applied uniformly across inlet and outlet boundaries in one flow direction while other boundaries are kept closed. We find the average relative permeabilities for the whole computational domain. It should be also noted that $\mu_{\mathrm{s}}$ can be arbitrary chosen with no impact on the average permeabilities, as long as Eq. 12 is strictly followed; this is also true for the magnitude of pressure drop applied at the boundaries of the system.

\section{Validation}

We here validate our percolation solver described previously in Sects. 5 and 6, by using a 2D model with a square grid and a 3D model with cubic grid blocks. The process simulated here is mainly for validation purposes and does not represent either conventional drainage or waterflooding. The main idea is to compute, using our solver, the site percolation thresholds and compare them to estimates from the literature of percolation theory. This only involves identifying trapped cells without accounting for trapped fluids inside them.

We use $1000 \times 1000$ grid cells for the $2 \mathrm{D}$ model, while $200 \times 200 \times 200$ grid cells are used for the 3D case. Figure 8 demonstrates the results, in which the fraction of invaded cells for an invading phase, $\eta_{\mathrm{w}}$ is plotted against the fraction of open cells for the invading phase, $\xi_{\mathrm{w}}$. The percolation threshold $\xi_{\mathrm{c}}$ is estimated at 0.59 and $0.31 \mathrm{in} 2 \mathrm{D}$ and $3 \mathrm{D}$ systems, respectively. These values are determined here precisely from the steady-state distribution of invaded cells $\psi_{\mathrm{w}}^{\infty}$ at the far boundary, as detailed in Sect. 5. From percolation theory for site percolation, $\xi_{\mathrm{c}}=0.5927$ in a 2D square lattice (Jacobsen 2015; Newman and Ziff 2000; 
Fig. 8 Fraction of invaded cells, $\eta_{\mathrm{w}}$ against fraction of open cells, $\xi_{\mathrm{w}}$ for $2 \mathrm{D}$ and $3 \mathrm{D}$ systems. For the $2 \mathrm{D}$ case, $1000 \times 1000$ grid cells have been used to produce the results, while using $200 \times 200 \times 200$ grid cells for the $3 \mathrm{D}$ case. The percolation threshold, $\xi_{\mathrm{c}}$, is estimated from this plot at 0.59 and 0.31 for site percolation on the $2 \mathrm{D}$ square lattice and the 3D simple cubic lattice, respectively. $\xi_{\mathrm{c}}$ is indicated in the plot by the sudden jump in $\eta_{\mathrm{w}}$, which is very clear in 2D while being less obvious in $3 \mathrm{D}$

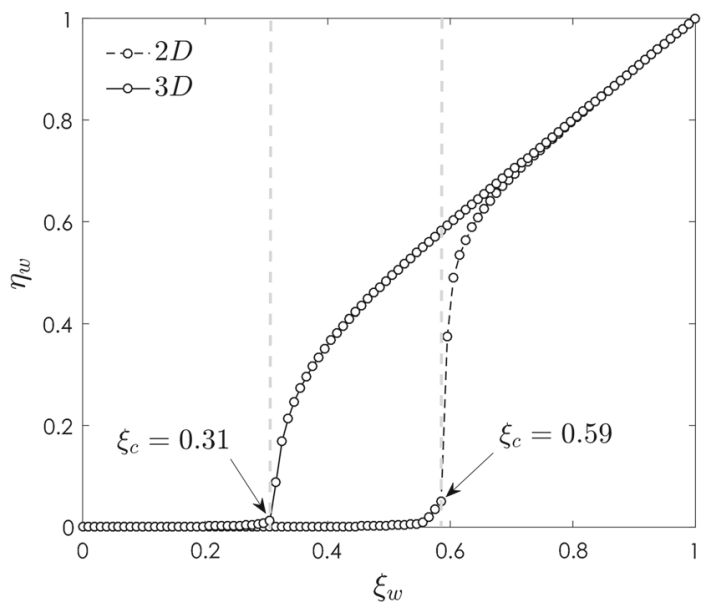

Lee 2007), and $\xi_{\mathrm{c}}=0.3116$ in a 3D simple cubic lattice (Xu et al. 2014; Škvor and Nezbeda 2009; Deng and Blöte 2005), which closely matches results obtained from our simulations. Higher accuracy can be achieved by increasing the number of grid cells and applying a very small step size in $\xi_{\mathrm{w}}$.

Results, as shown in Fig. 8, indicate a strong correlation between the percolation threshold, $\xi_{\mathrm{c}}$, and the dimensionality of the model. This is usually expressed in terms of the coordination number; the number of nearest neighbouring grid cells, being 4 in the 2D and 6 in the 3D models used here. As the coordination number increases, the percolation threshold decreases, making lattice representation for macroscopic percolations a very critical issue for 2D and 3D models, since they can be represented with different combinations of coordination numbers. For this, it is not obvious which coordination number should be used for a valid representation of a heterogeneous system. Nevertheless, it has been shown that these sensitivities to coordination number become less important in spatially correlated media, where cells with similar properties are clustered together (Ioannidis et al. 1993, 1996). Hence, it is less likely to have an accessible cell neighbouring a trapped cell. This, however, depends on the correlation length of the system under investigation (Ioannidis et al. 1996).

Figure 9 shows spatial distributions of $\boldsymbol{\gamma}_{\mathbf{w}}$ and $\boldsymbol{\psi}_{\mathbf{w}}^{\infty}$ for the 2D system at different values of $\xi_{\mathrm{w}}$. The process starts from the left face in the horizontal direction, while boundaries on the vertical direction are closed. Blue here represents cells in which an invading phase resides (invaded cells), while other cells are trapped. These trapped regions contain two types of cell: (1) cells with local trapping of the invading phase (green), and (2) cells that are accessible by the invading phase but are trapped at the large scale as being locked in by the green cells (grey).

Figure 9a shows the stationary distribution at $\eta_{\mathrm{w}}=2.64 \%$ and $\xi_{\mathrm{w}}=57.52 \%$, where no global connectivity is observed. In Fig. 9b, simulations are shown for just below $\xi_{\mathrm{c}}$ at $\eta_{\mathrm{w}}=19.05 \%$ and $\xi_{\mathrm{w}}=59.32 \%$. Figure 9c indicates invasion percolation just above $\xi_{\mathrm{c}}$ at $\eta_{\mathrm{w}}=41.53 \%$ and $\xi_{\mathrm{w}}=59.42 \%$. Lastly, Fig. $9 \mathrm{~d}$ shows stationary distributions at $\eta_{\mathrm{w}}=50.15 \%$ and $\xi_{\mathrm{w}}=60.51 \%$, above $\xi_{\mathrm{c}}$.

Similarly for the simple cubic model, Fig. 10 depicts large-scale invasion percolation in a $3 \mathrm{D}$ system. The invading phase is allowed to enter the system from one end while the opposite end is left open. The other boundaries are closed. Invaded cells are coloured based on their distance from the inlet boundary. Other cells are not displayed. Figure 10a shows 


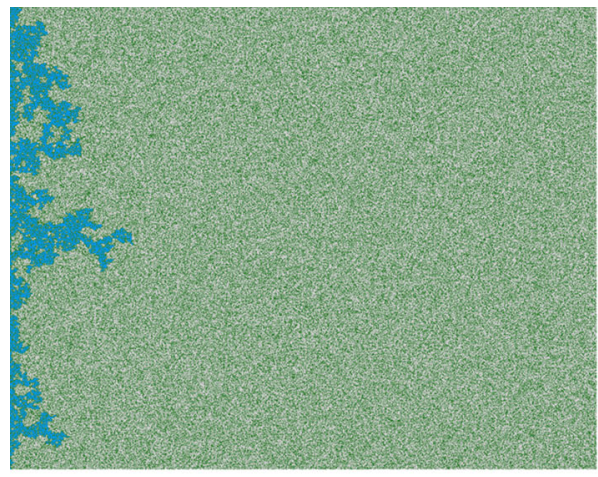

(a)

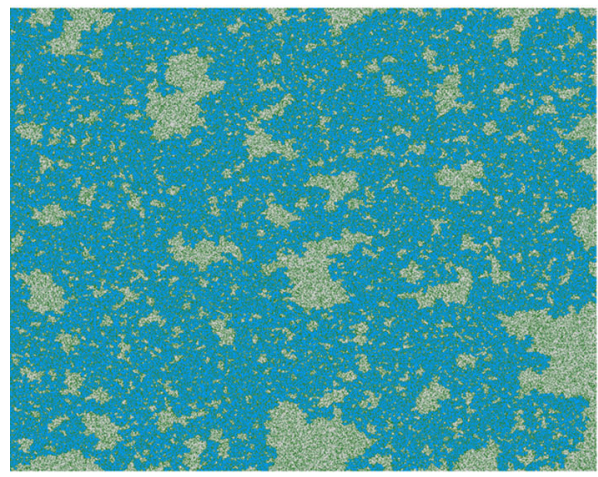

(c)

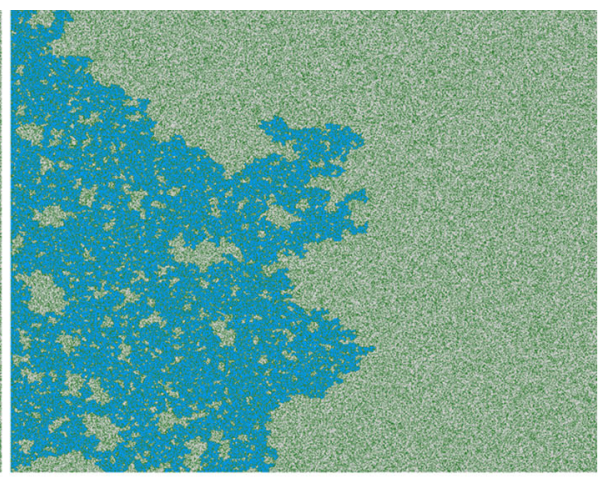

(b)

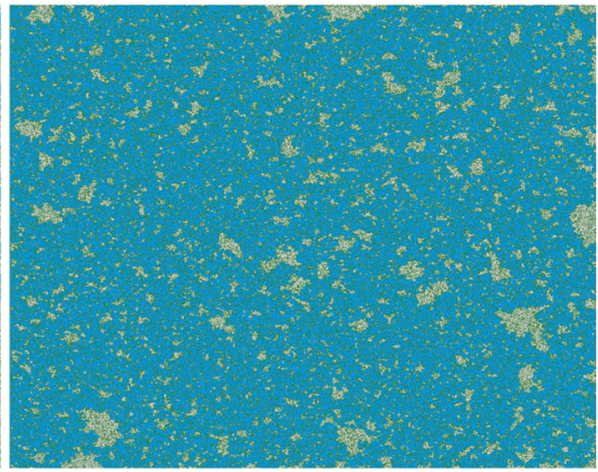

(d)

Fig. 9 Site percolation on a square lattice consisting of $1000 \times 1000$ grid cells. The process starts from the left face in the horizontal direction, while boundaries on the vertical direction are closed. Blue represents invaded cells while other cells are trapped. The trapped regions contain two types of cells: (1) green cells indicating that they do not percolate the invading phase across them (local trapping of the invading phase) and (2) grey cells representing cells with large-scale trapping; they are accessible by the invading phase but are surrounded by green cells. a The stationary distribution of invaded cells (blue) at a given capillary equilibrium state below the percolation threshold where there is no global connectivity $\left(\eta_{\mathrm{w}}=2.64 \%, \xi_{\mathrm{w}}=57.52 \%\right)$. b The fluid distribution just below $\xi_{\mathrm{c}}$ at $\eta_{\mathrm{W}}=19.05 \%$ and $\xi_{\mathrm{W}}=59.32 \%$. $\mathbf{c}$ Invasion percolation just above $\xi_{\mathrm{c}}$ at $\eta_{\mathrm{w}}=41.53 \%$ and $\xi_{\mathrm{w}}=59.42 \%$. d The distribution for at $\eta_{\mathrm{w}}=50.15 \%$ and $\xi_{\mathrm{w}}=60.51 \%$

the stationary distribution of invaded cells below $\xi_{\mathrm{c}}$ at $\eta_{\mathrm{w}}=1.68 \%, \xi_{\mathrm{w}}=30.70 \%$, whereas Fig. 10b displays simulation just below $\xi_{\mathrm{c}}$ at $\eta_{\mathrm{w}}=2.50 \%$ and $\xi_{\mathrm{w}}=30.89 \%$. In Fig. 10c, invasion percolation just above $\xi_{\mathrm{c}}$ at $\eta_{\mathrm{w}}=4.48 \%$ and $\xi_{\mathrm{w}}=31.13 \%$ is demonstrated. Figure $10 \mathrm{~d}$ shows stationary distributions at $\eta_{\mathrm{w}}=6.74 \%$ and $\xi_{\mathrm{w}}=31.36 \%$, well above $\xi_{\mathrm{c}}$.

\section{Illustrative Examples}

We present here an example solution using our large-scale invasion percolation algorithm coupled with a Darcy-scale solver. We do not consider hysteresis in the capillary pressure and relative permeability: locally trapped saturations are not allowed to change with initial 


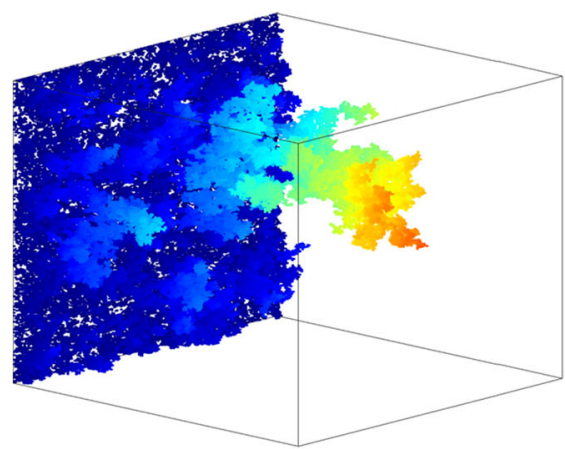

(a)

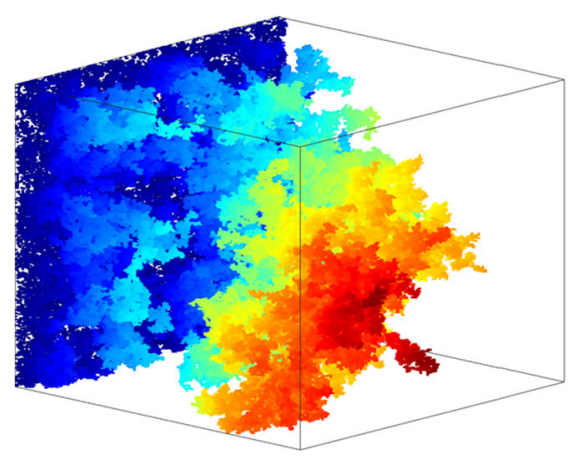

(c)

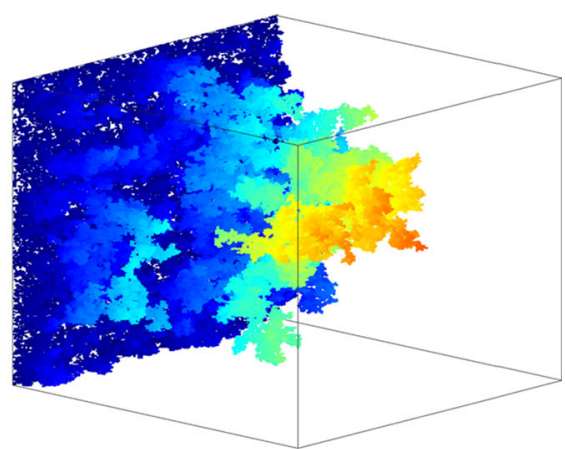

(b)

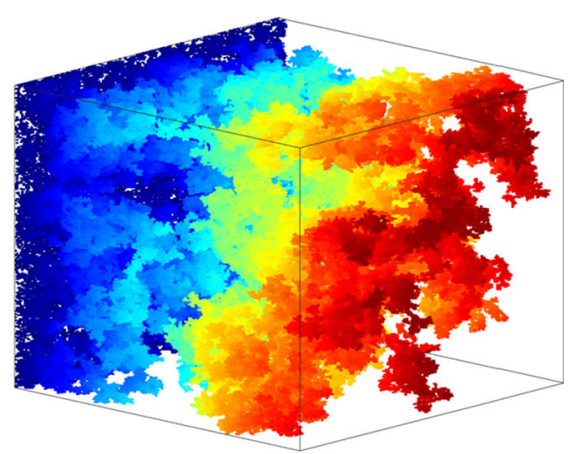

(d)

Fig. 10 Invasion percolation on a simple cubic lattice with $200 \times 200 \times 200$ grid cells. Two opposite boundary faces are open, while the other faces are closed. Coloured cells represent the stationary distribution of invaded cells at a given capillary equilibrium state. The change in colours indicates distance from inlet (blue being close to the inlet boundary). a The stationary distribution of invaded cells below $\xi_{\mathrm{c}}$ at $\eta_{\mathrm{W}}=1.68 \%, \xi_{\mathrm{W}}=30.70 \%$. b The distribution just below $\xi_{\mathrm{c}}$ at $\eta_{\mathrm{W}}=2.50 \%$ and $\xi_{\mathrm{W}}=30.89 \%$. c Invasion percolation just above $\xi_{\mathrm{c}}$ at $\eta_{\mathrm{W}}=4.48 \%$ and $\xi_{\mathrm{W}}=31.13 \%$. d The distributions for $\eta_{\mathrm{W}}=6.74 \%$ and $\xi_{\mathrm{W}}=31.36 \%$

saturation. This will be a topic for future work. For our simulations, we use two models: a $2 \mathrm{D}$ with $1000 \times 1000$ grid cells, and another a 3D model with $200 \times 200 \times 200$ grid cells.

We use synthetic data representing four different wettability states: strongly water-wet (SWW), weakly water-wet (WWW), mixed-wet (MW) and oil-wet (OW). For each wetting state, absolute permeability is randomly distributed from an uncorrelated log-normal distribution with a known mean and standard deviation. The mean is kept fixed in all simulations at $\mu_{\ln k}=-29.9336$, equivalent to $\mu_{k}=1 \times 10^{-13} \mathrm{~m}^{2}$, while the standard deviation varies over two sets of values $\sigma_{\ln k}=\{1,2\}$. Porosity is kept constant at $\phi=0.25$.

For the modelling of multiphase flow functions, the following mathematical expressions are used:

$$
p_{\mathrm{c}}^{\mathrm{dr}}\left(S_{\mathrm{w}}\right)= \begin{cases}\left(p_{\mathrm{c}}^{\max }-p_{\mathrm{e}}\right)\left(1-\frac{S_{\mathrm{w}}-S_{\mathrm{wir}}}{1-S_{\mathrm{wir}}}\right)^{n_{\mathrm{c}}^{\mathrm{dr}}}+p_{\mathrm{e}}, & S_{\mathrm{w}} \in\left[S_{\mathrm{wir}}, 1\right] \\ \varnothing, & S_{\mathrm{w}} \notin\left[S_{\text {wir }}, 1\right]\end{cases}
$$


Table 1 Parameters used to generate relative permeability and capillary pressure data in the Illustrative Examples section

\begin{tabular}{lllll}
\hline & Strongly water-wet & Weakly water-wet & Mixed-wet & Oil-wet \\
\hline$S_{\text {wir }}$ & 0.1 & 0.1 & 0.1 & 0.1 \\
$S_{\mathrm{orw}}$ & 0.4 & 0.3 & 0.15 & 0.05 \\
$k_{\mathrm{rW}}^{\max }$ & 0.1 & 0.2 & 0.5 & 0.95 \\
$n_{\mathrm{w}}$ & 2 & 2 & 8 & 1.5 \\
$k_{\mathrm{ro}}^{\max }$ & 1 & 1 & 1 & 1 \\
$n_{\mathrm{O}}$ & 1 & 1.5 & 2.5 & 4 \\
$p_{\mathrm{c}}^{\max }(\mathrm{kPa})$ & 150 & 150 & 150 & 150 \\
$n_{\mathrm{c}}^{+}$ & 2 & 1.7 & 2 & $\varnothing$ \\
$p_{\mathrm{c}}^{\min }(\mathrm{kPa})$ & 0 & -50 & -100 & -150 \\
$n_{\mathrm{c}}^{-}$ & $\varnothing$ & 1.5 & 2.5 & 3 \\
$S^{*}$ & 0.6 & 0.55 & 0.5 & 0.1 \\
$p_{\mathrm{e}}(\mathrm{kPa})$ & 1 & 1 & 1 & 1 \\
$n_{\mathrm{c}}^{\mathrm{dr}}$ & 2 & 2 & 2 & 2 \\
\hline
\end{tabular}

Equations 15 and 16 are used for capillary pressures for drainage and waterflooding, respectively, while using Eqs. 17 and 18 for water and oil relative permeabilities, respectively. (Modified from Blunt (2017))

Table 2 Base parameters used in the J-function calculations

\begin{tabular}{ll}
\hline$k_{\mathrm{b}}, \mathrm{m}^{2}$ & $1 \times 10^{-13}$ \\
$\phi_{\mathrm{b}}$, fraction & 0.25 \\
$\sigma_{\mathrm{wo}} \cos \theta_{\mathrm{wo}}, \mathrm{N} / \mathrm{m}$ & $3 \times 10^{-2}$ \\
\hline
\end{tabular}

$$
\begin{aligned}
p_{\mathrm{c}}\left(S_{\mathrm{w}}\right) & =\left\{\begin{array}{lc}
p_{\mathrm{c}}^{\max }\left(1-\frac{S_{\mathrm{w}}-S_{\mathrm{wir}}}{S^{*}-S_{\mathrm{wir}}}\right)^{n_{\mathrm{c}}^{+}}, & S_{\mathrm{w}} \in\left[S_{\mathrm{wir}}, S^{*}\right] \\
p_{\mathrm{c}}^{\min }\left(\frac{S_{\mathrm{w}}-S^{*}}{1-S^{*}-S_{\text {orw }}}\right)^{n_{\mathrm{c}}^{-}}, & S_{\mathrm{w}} \in\left[S^{*}, 1-S_{\text {orw }}\right]
\end{array}\right. \\
k_{\mathrm{rw}}\left(S_{\mathrm{w}}\right) & =\left\{\begin{array}{lc}
k_{\mathrm{rw}}^{\max }\left(\frac{S_{\mathrm{w}}-S_{\text {wir }}}{1-S_{\text {wir }}-S_{\text {orw }}}\right)^{n_{\mathrm{w}}}, & S_{\mathrm{w}} \in\left[S_{\text {wir }}, 1-S_{\text {orw }}\right] \\
\varnothing, & S_{\mathrm{w}} \notin\left[S_{\mathrm{wir}}, 1-S_{\text {orw }}\right]
\end{array}\right. \\
k_{\mathrm{ro}}\left(S_{\mathrm{w}}\right) & = \begin{cases}k_{\mathrm{ro}}^{\max }\left(1-\frac{S_{\mathrm{w}}-S_{\mathrm{wir}}}{1-S_{\text {wir }}-S_{\text {orw }}}\right)^{n_{\mathrm{o}}}, & S_{\mathrm{w}} \in\left[S_{\text {wir }}, 1-S_{\text {orw }}\right] \\
\varnothing, & S_{\mathrm{w}} \notin\left[S_{\text {wir }}, 1-S_{\text {orw }}\right]\end{cases}
\end{aligned}
$$

where Eqs. 15 and 16 are used for capillary pressures for drainage $p_{\mathrm{c}}^{\mathrm{dr}}\left(S_{\mathrm{W}}\right)$ and waterflooding $p_{\mathrm{c}}\left(S_{\mathrm{w}}\right)$, respectively, while Eqs. 17 and 18 are used for water $k_{\mathrm{rw}}\left(S_{\mathrm{w}}\right)$ and oil $k_{\mathrm{ro}}\left(S_{\mathrm{w}}\right)$ relative permeabilities, respectively; $p_{\mathrm{c}}^{\max }$ is the maximum capillary pressure in $\mathrm{Pa}, p_{\mathrm{c}}^{\min }$ is the minimum capillary pressure in $\mathrm{Pa}, p_{\mathrm{e}}$ is the entry pressure in $\mathrm{Pa}, S^{*}$ is the water saturation specified at zero capillary pressure, $n_{\mathrm{c}}^{\mathrm{dr}}$ is the exponent used in the drainage capillary pressure, $n_{\mathrm{c}}^{+}$and $n_{\mathrm{c}}^{-}$are the exponents used in the positive and negative regions of the capillary pressure for waterflooding, respectively, $n_{\mathrm{w}}$ and $n_{\mathrm{o}}$ are the exponents used in the relative permeability functions for water and oil, respectively, $k_{\mathrm{rw}}^{\max }$ is the maximum relative permeability to water, and $k_{\mathrm{ro}}^{\max }$ is the maximum relative permeability to oil. J-function scaling Leverett (1941) is used to correlate capillary pressures with rock properties, resulting in the following expression: 


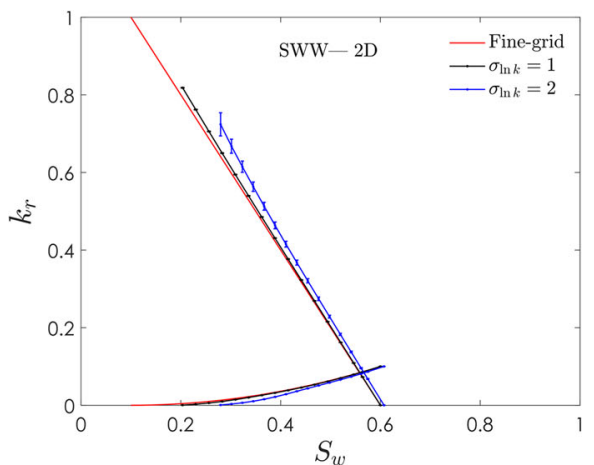

(a)

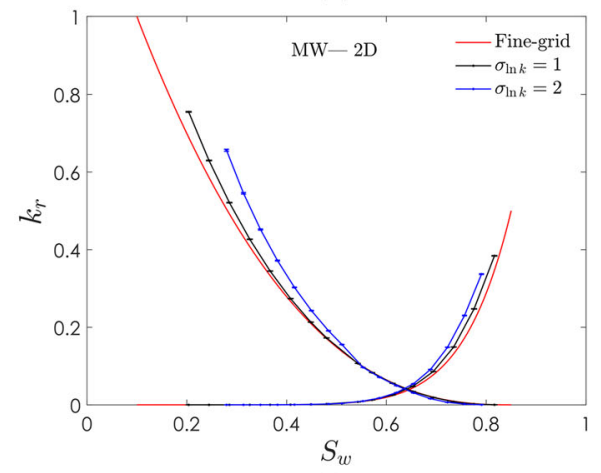

(c)

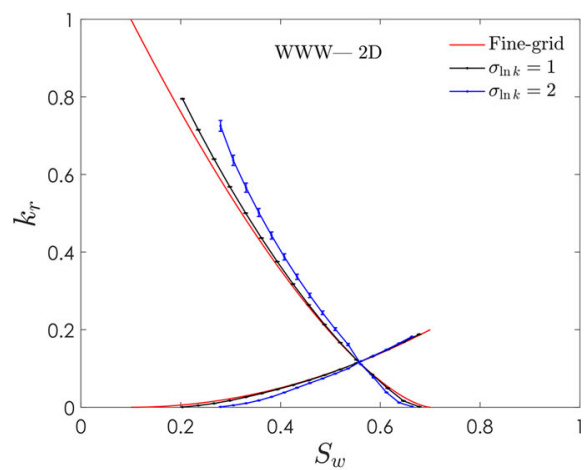

(b)

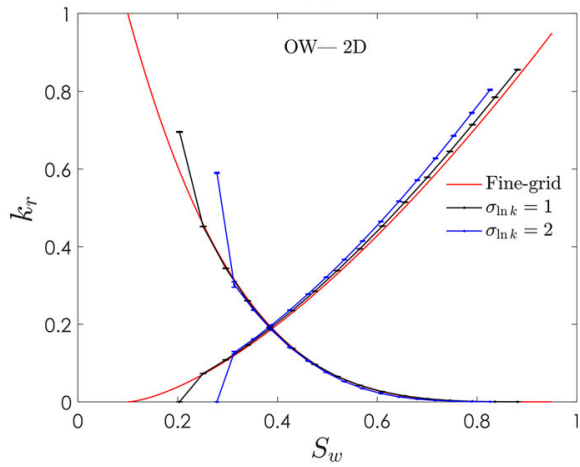

(d)

Fig. 11 Upscaled relative permeabilities in 2D for capillary-controlled displacements including the impact of percolation for different wettability states and permeability distributions with $\sigma_{\ln k}=\{1,2\}$. a $k_{r}$ data in strongly water-wet; $\mathbf{b}$ weakly water-wet; $\mathbf{c}$ mixed-wet; and $\mathbf{d}$ oil-wet systems. The original fine-grid $k_{r}$ curves are also included for comparison purposes. The curves are plotted using average values from five simulations, while the bars represent their standard deviations

$$
p_{\mathrm{c}_{i}}\left(S_{\mathrm{w}}\right)=\frac{\sqrt{k_{\mathrm{b}} / \phi_{\mathrm{b}}}}{\sqrt{k_{i} / \phi_{i}}} p_{\mathrm{c}_{\mathrm{b}}}\left(S_{\mathrm{w}}\right)
$$

where $p_{\mathrm{c}_{i}}$ is the capillary pressure at cell $i$ in $\mathrm{Pa}, p_{\mathrm{c}_{\mathrm{b}}}$ is a base capillary pressure measured in the laboratory in $\mathrm{Pa}, k_{i}$ and $\phi_{i}$ are absolute permeability and porosity in cell $i$ in $\mathrm{m}^{2}$ and fraction, respectively, and $k_{\mathrm{b}}$ and $\phi_{\mathrm{b}}$ are base permeability and porosity, respectively.

All model parameters for multiphase flow calculations are listed in Table 1, while Table 2 lists the base parameters used in the J-function calculations, for both drainage and waterflooding. The parameters $k_{\mathrm{b}}$ and $\phi_{\mathrm{b}}$ are used as a reference for the J-function calculations. In a normal practice, these values are obtained from the same rock where the capillary pressure measurements $p_{\mathrm{cb}}$ were taken from. The main purpose of the J-function scaling is to extrapolate capillary pressure information for systems with known basic properties, e.g. $k_{i}$ and $\phi_{i}$, for which capillary pressure data are originally unknown. For each wettability state, Eq. 19 is applied to generate a capillary pressure curve in each and every cell in our simulation model.

We now perform simulations to determine average quantities as previously described. For each wetting state, five simulations are performed for each $\sigma_{\ln k}$. The computations of average relative permeabilities are performed at 16 capillary equilibrium states. The percolation simulations, on the other hand, are performed at a much more refined $p_{\mathbf{c}}^{\text {level }}$ distribution, 


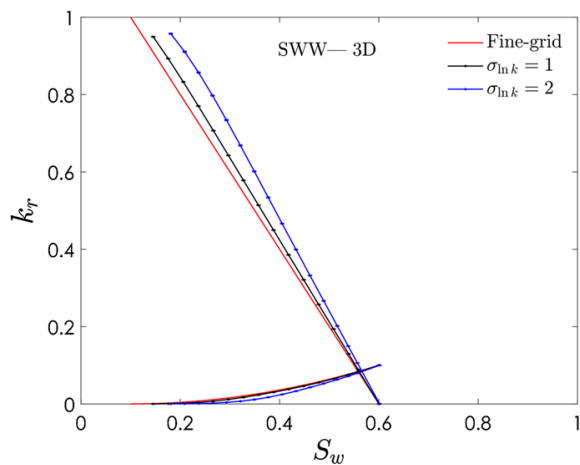

(a)

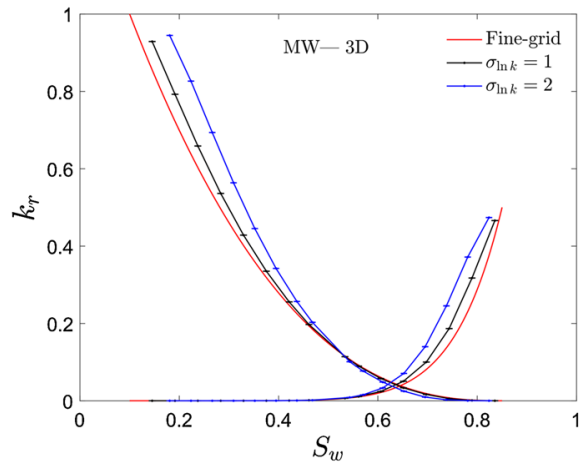

(c)

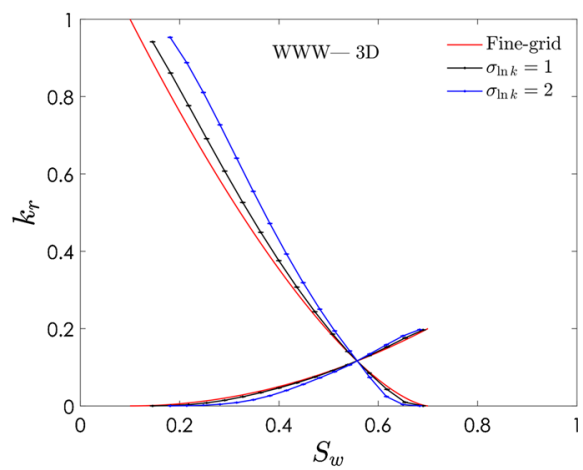

(b)

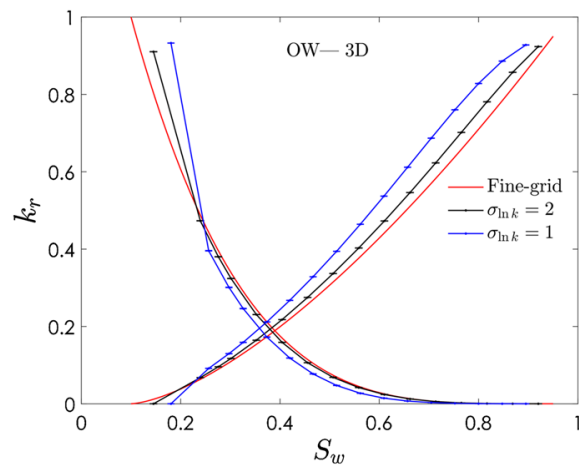

(d)

Fig. 12 Upscaled relative permeabilities in 3D for capillary-controlled displacements including the impact of percolation for different wettability states and permeability distributions with $\sigma_{\ln k}=\{1,2\}$. a $k_{r}$ curves in strongly water-wet; $\mathbf{b}$ weakly water-wet; $\mathbf{c}$ mixed-wet; and $\mathbf{d}$ oil-wet systems. Original $k_{r}$ curves are also included for comparison purposes. The curves are plotted using average values from five simulations, while the bars represent their standard deviations

estimated from $\boldsymbol{p}_{\mathbf{c}}^{\max }$ and $\boldsymbol{p}_{\mathbf{c}}^{\min }$, to identify accurately trapping, from which average water saturations are determined. The elements of $\boldsymbol{p}_{\mathbf{c}}^{\mathbf{m a x}}$ and $\boldsymbol{p}_{\mathbf{c}}^{\min }$ distributions can be calculated from Eq. 19 as follows:

$$
\begin{aligned}
& p_{\mathrm{c}_{i}}^{\max }=\frac{\sqrt{k_{\mathrm{b}} / \phi_{\mathrm{b}}}}{\sqrt{k_{i} / \phi_{i}}} p_{\mathrm{c}}^{\max } \\
& p_{\mathrm{c}_{i}}^{\min }=\frac{\sqrt{k_{\mathrm{b}} / \phi_{\mathrm{b}}}}{\sqrt{k_{i} / \phi_{i}}} p_{\mathrm{c}}^{\text {min }}
\end{aligned}
$$

where $p_{\mathrm{c}_{i}}^{\max }$ and $p_{\mathrm{c}_{i}}^{\min }$ are the maximum and minimum capillary pressures at cell $i, p_{\mathrm{c}}^{\max }$ and $p_{\mathrm{c}}^{\mathrm{min}}$ are given for all wetting conditions in Table 1. Other parameters are defined previously in Eq. 19. Repeated elements are removed from the distributions and sorted. The $\boldsymbol{p}_{\mathbf{c}}^{\text {level }}$ distribution is then estimated by computing percentiles at every half a percentage point.

Figures 11 and 12 show the upscaled relative permeabilities for $\sigma_{\ln k}=\{1,2\}$, for $2 \mathrm{D}$ and $3 \mathrm{D}$, respectively. Each curve represents the overall average of the five simulations, while the bars indicate their standard deviations. We can observe, in general, that as $\sigma_{\ln k}$ increases, the deviations of both $\tilde{k}_{\text {rw }}$ and $\tilde{k}_{\text {ro }}$ from the fine-grid functions become more apparent. It is also 


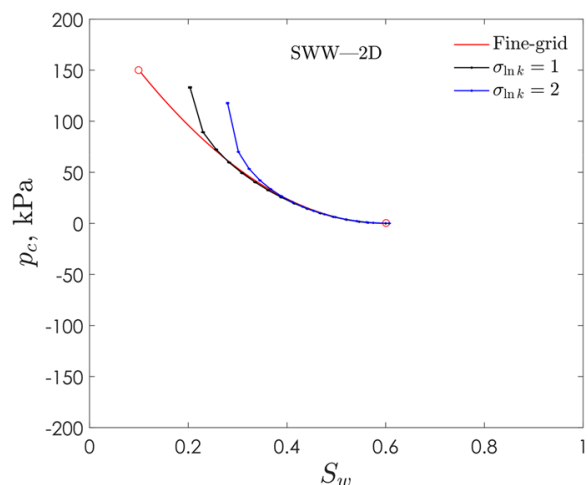

(a)

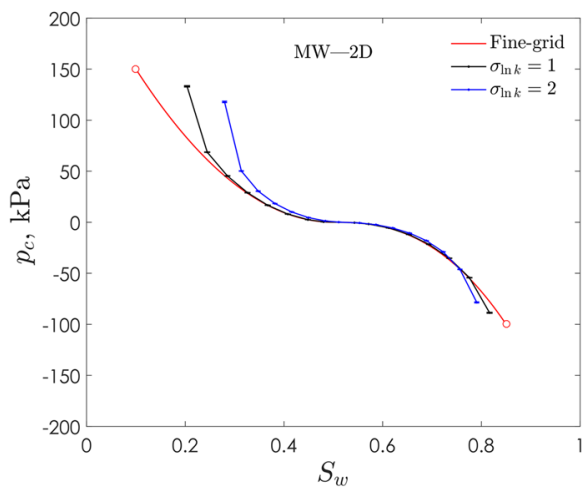

(c)

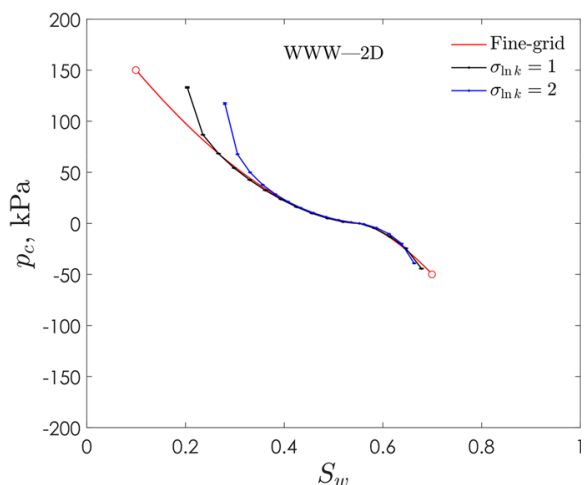

(b)

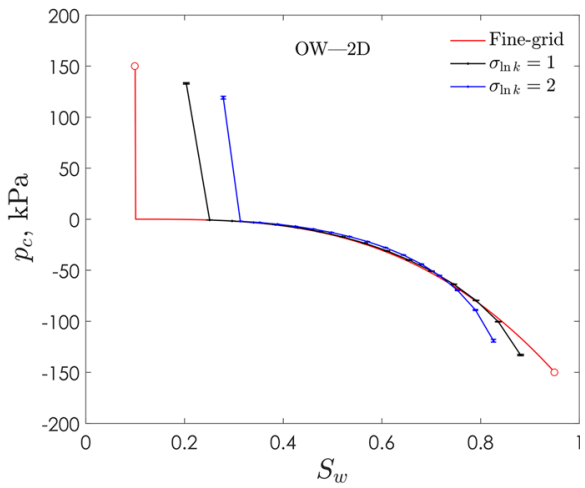

(d)

Fig. 13 Upscaled capillary pressures in 2D for capillary-controlled displacements for different wettability states and permeability distributions with $\sigma_{\ln k}=\{1,2\}$. a $p_{\mathrm{c}}$ curves in strongly water-wet; $\mathbf{b}$ weakly waterwet; c mixed-wet; and d oil-wet media. Original $p_{\mathrm{c}}$ curves are also included for comparison purposes. The curves are plotted using average values from five simulations, while the bars represent their standard deviations

shown that the trapping of fluids also increases with $\sigma_{\ln k}$, though the trapping in $2 \mathrm{D}$ is larger than in 3D. We also see in Fig. 11 that the maximum value of $\tilde{k}_{\text {rw }}$ and $\tilde{k}_{\text {ro }}$ decreases with more trapping, indicating that the additional trapped fluids preferentially fill some regions of the domain that have some contribution to the overall permeability; this is only true for $2 \mathrm{D}$. In $3 \mathrm{D}$, however, this is not observed (Fig. 12), suggesting that the additional trapped fluids occupy regions that do not contribute to the overall permeability of the sample.

The averaged water relative permeabilities are only finite once the water percolates across the system, which occurs at a finite occupancy. We see an increase in the minimum water saturation at which water flows across the domain. At the end of primary drainage, water is trapped at the large scale in isolated clusters distributed across the whole domain. As water is injected into the system, these clusters reconnect increasing the averaged water relative permeabilities more rapidly.

It is demonstrated that $\tilde{k}_{\text {ro }}$ generally increases over a wide range of $\tilde{S}_{\mathrm{W}}$ as $\sigma_{\ln k}$ increases, especially for SWW, WWW and MW media. This indicates that water preferentially resides in the lower permeability rock at a given $\tilde{S}_{\mathrm{w}}$. Similarly for $\tilde{k}_{\mathrm{rw}}$ in MW and OW systems, 


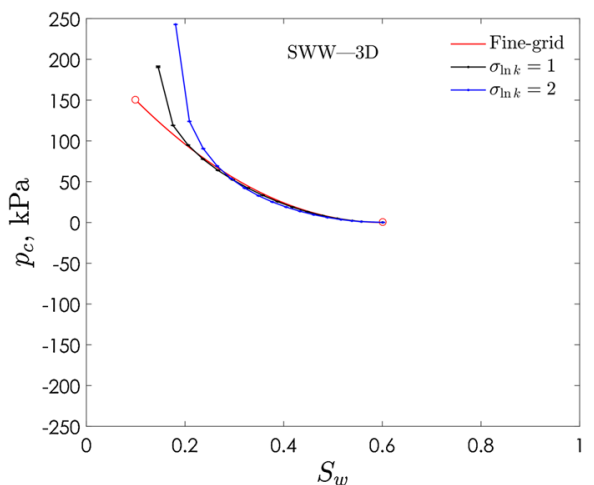

(a)

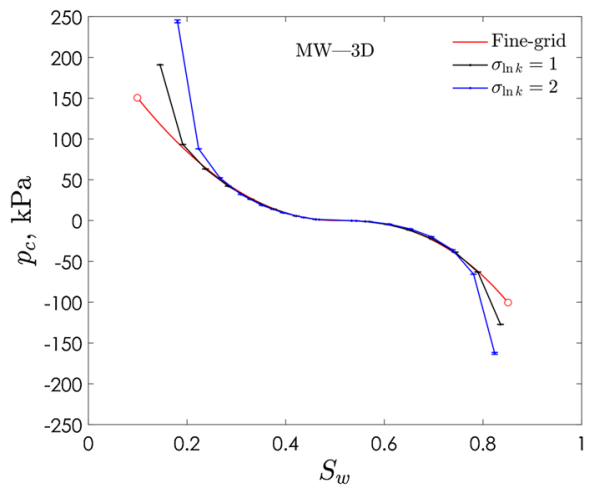

(c)

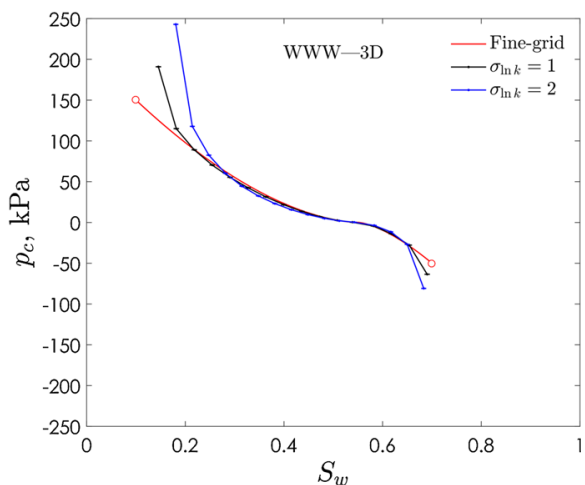

(b)

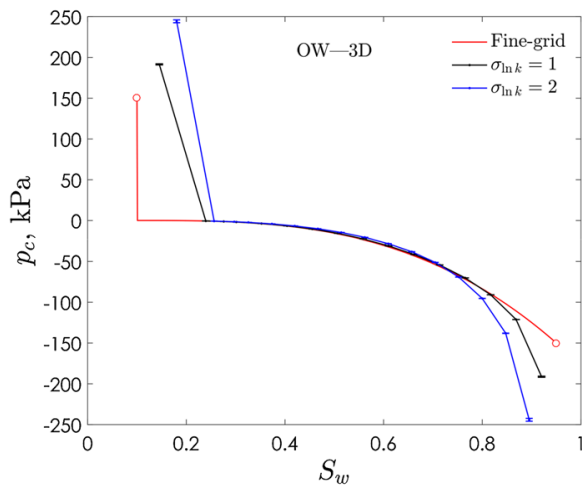

(d)

Fig. 14 Upscaled capillary pressures in 3D for capillary-controlled displacements for different wettability states and permeability distributions with $\sigma_{\ln k}=\{1,2\}$. a $p_{\mathrm{c}}$ curves in strongly water-wet; $\mathbf{b}$ weakly waterwet; c mixed-wet; and d oil-wet media. Original $p_{\mathrm{c}}$ curves are also included for comparison purposes. The curves are plotted using average values from five simulations, while the bars represent their standard deviations

where it generally increases for the late values of $\tilde{S}_{\mathrm{w}}$ as $\sigma_{\ln k}$ increases, suggesting that the remaining oil now tends to occupy the lower permeability portions of the system.

Average capillary pressures are shown in Figs. 13 and 14 for 2D and 3D models, respectively. In general, little sensitivity is observed on the trends of the curves for all the wetting states and permeability distributions, except near the end-point saturations. What is observed is the trapping of fluids as shown in the figures and listed in Tables 3 and 4, for 2D and 3D systems, respectively. These values reported in the table are derived from the distributions generated from the five simulation runs. The symbols $\tilde{S}_{\text {wir }}$ and $\tilde{S}_{\text {orw }}$ are used to refer to the upscaled irreducible water and residual oil saturations, respectively, while $\left|\Delta S_{\text {wir }}\right|$ and $\left|\Delta S_{\text {orw }}\right|$ show the absolute difference between the upscaled quantities and the fine-grid inputs. As can be seen that more fluids are trapped at the Darcy scale as $\sigma_{\ln k}$ increases.

We observe also that the trapping of water $\tilde{S}_{\text {wir }}$ does not vary between the different wetting states, but with $\sigma_{\ln k}$ and the dimensionality of the system. This is because the capillary pressure function defined for drainage being the same for all the wetting states. On average, about $10 \%$ of the water is trapped at the large scale in $2 \mathrm{D}$ for $\sigma_{\ln k}=1$, and $18 \%$ for $\sigma_{\ln k}=2$, while about half of that is seen in $3 \mathrm{D}$. 
Table 3 Upscaled end-point saturations for the 2D model (1 million grid cells)

\begin{tabular}{rllll}
\hline & $\tilde{S}_{\text {Wir }}$ & $\tilde{S}_{\text {orw }}$ & $\left|\Delta S_{\text {Wir }}\right|$ & $\left|\Delta S_{\text {orw }}\right|$ \\
\hline SWW & & & & \\
$\sigma_{\ln k}=1$ & $0.2036 \pm 0.0013$ & $0.4000 \pm 0.0000$ & $0.1036 \pm 0.0013$ & $0.0000 \pm 0.0000$ \\
$\sigma_{\ln k}=2$ & $0.2797 \pm 0.0004$ & $0.3928 \pm 0.0001$ & $0.1797 \pm 0.0004$ & $0.0072 \pm 0.0001$ \\
$\mathrm{WWW}$ & & & & \\
$\sigma_{\ln k}=1$ & $0.2036 \pm 0.0004$ & $0.3221 \pm 0.0005$ & $0.1036 \pm 0.0004$ & $0.0221 \pm 0.0005$ \\
$\sigma_{\ln k}=2$ & $0.2798 \pm 0.0008$ & $0.3368 \pm 0.0006$ & $0.1798 \pm 0.0008$ & $0.0368 \pm 0.0006$ \\
$\mathrm{MW}$ & & & & \\
$\sigma_{\ln k}=1$ & $0.2036 \pm 0.0013$ & $0.1835 \pm 0.0006$ & $0.1036 \pm 0.0013$ & $0.0335 \pm 0.0006$ \\
$\sigma_{\ln k}=2$ & $0.2796 \pm 0.0016$ & $0.2094 \pm 0.0007$ & $0.1796 \pm 0.0016$ & $0.0594 \pm 0.0007$ \\
OW & & & & \\
$\sigma_{\ln k}=1$ & $0.2036 \pm 0.0011$ & $0.1191 \pm 0.0014$ & $0.1036 \pm 0.0011$ & $0.0691 \pm 0.0014$ \\
$\sigma_{\ln k}=2$ & $0.2785 \pm 0.0021$ & $0.1741 \pm 0.0029$ & $0.1785 \pm 0.0021$ & $0.1241 \pm 0.0029$ \\
\hline$\Delta S_{\mathrm{Win}}$ & & & &
\end{tabular}

$\left|\Delta S_{\text {wir }}\right|$ and $\left|\Delta S_{\text {orw }}\right|$ refer to the absolute difference between the upscaled quantities and the fine-grid inputs

Table 4 Upscaled end-point saturations for the 3D model (8 million grid cells)

\begin{tabular}{rllll}
\hline & $\tilde{S}_{\text {wir }}$ & $\tilde{S}_{\text {orw }}$ & $\left|\Delta S_{\text {wir }}\right|$ & $\left|\Delta S_{\text {orw }}\right|$ \\
\hline $\mathrm{SWW}$ & & & & \\
$\sigma_{\ln k}=1$ & $0.1454 \pm 0.0002$ & $0.4000 \pm 0.0000$ & $0.0454 \pm 0.0002$ & $0.0000 \pm 0.0000$ \\
$\sigma_{\ln k}=2$ & $0.1808 \pm 0.0003$ & $0.3984 \pm 0.0000$ & $0.0808 \pm 0.0003$ & $0.0016 \pm 0.0000$ \\
$\mathrm{WWW}$ & & & & \\
$\sigma_{\ln k}=1$ & $0.1454 \pm 0.0001$ & $0.3097 \pm 0.0000$ & $0.0454 \pm 0.0001$ & $0.0097 \pm 0.0000$ \\
$\sigma_{\ln k}=2$ & $0.1808 \pm 0.0001$ & $0.3167 \pm 0.0000$ & $0.0808 \pm 0.0001$ & $0.0167 \pm 0.0000$ \\
$\mathrm{MW}$ & & & & \\
$\sigma_{\ln k}=1$ & $0.1454 \pm 0.0001$ & $0.1645 \pm 0.0000$ & $0.0454 \pm 0.0001$ & $0.0145 \pm 0.0000$ \\
$\sigma_{\ln k}=2$ & $0.1807 \pm 0.0003$ & $0.1763 \pm 0.0003$ & $0.0807 \pm 0.0003$ & $0.0263 \pm 0.0003$ \\
$\mathrm{OW}$ & & & & \\
$\sigma_{\ln k}=1$ & $0.1454 \pm 0.0001$ & $0.0798 \pm 0.0003$ & $0.0454 \pm 0.0001$ & $0.0298 \pm 0.0003$ \\
$\sigma_{\ln k}=2$ & $0.1805 \pm 0.0002$ & $0.1049 \pm 0.0004$ & $0.0805 \pm 0.0002$ & $0.0549 \pm 0.0004$ \\
\hline
\end{tabular}

$\left|\Delta S_{\text {wir }}\right|$ and $\left|\Delta S_{\text {orw }}\right|$ are the absolute difference between the upscaled quantities and the fine-grid inputs

For the large-scale trapping of oil, we observe different behaviours than water between the wetting conditions, influenced by the capillary pressure functions for waterflooding. Here, the highest trapping happens in oil-wet media at $12 \%$ and $5 \%$ in $2 \mathrm{D}$ and $3 \mathrm{D}$, respectively, at $\sigma_{\ln k}=2$. This trapping is in addition to the pore-scale trapping, given by $S_{\text {orw }}$ in Table 1 .

\section{Conclusions}

A modified upscaling procedure for steady-state capillary-controlled immiscible displacements is proposed, which accounts for the large-scale connectivity in Darcy-scale simulations. The key idea is that at a given capillary equilibrium state, some regions in the system may 
form disconnected clusters preventing global connectivity. To tackle such displacements, a revised steady-state upscaling algorithm is introduced accounting for the trapping of fluids in a large-scale invasion percolation displacement. The process is explained through illustrative examples, in which four different wettability states are studied-strongly water-wet, weakly water-wet, mixed-wet and oil-wet. It is observed that, at Darcy scale, the capillary trapping for water increases in heterogeneous media and is influenced mainly by the capillary pressure function defined for drainage. For the large-scale trapping of oil, we observe different behaviours between the wetting conditions, controlled by the capillary pressure functions for waterflooding, in which the highest trapping is observed in oil-wet systems.

The analysis of this paper could be extended in future studies to explore the impact of viscous forces, using realistic geology, and including the effects of hysteresis in the multiphase flow functions.

Acknowledgements The first author would like to thank Saudi Aramco for their generous financial support towards his $\mathrm{PhD}$ at Imperial College London.

Open Access This article is distributed under the terms of the Creative Commons Attribution 4.0 International License (http://creativecommons.org/licenses/by/4.0/), which permits unrestricted use, distribution, and reproduction in any medium, provided you give appropriate credit to the original author(s) and the source, provide a link to the Creative Commons license, and indicate if changes were made.

\section{References}

Barker, J.W., Dupouy, P.: An analysis of dynamic pseudo-relative permeability methods for oil-water flows. Petrol. Geosci. 5(4), 385-394 (1999)

Barker, J.W., Thibeau, S.: A critical review of the use of pseudorelative permeabilities for upscaling. SPE J. 12(2), 138-143 (1997)

Blunt, M.J.: Multiphase Flow in Permeable Media: a Pore-Ccale Perspective. Cambridge University Press, Cambridge (2017)

Braun, E.M., Blackwell, R.J.: A steady-state technique for measuring oil-water relative permeability curves at reservoir conditions. In: SPE Annual Technical Conference and Exhibition, SPE-10155-MS, pp. 1-10 (1981)

Coll, C., Muggeridge, A.H., Jing, X.D.: Regional upscaling: a new method to upscale waterflooding in heterogeneous reservoirs for a range of capillary and gravity effects. SPE J. 6(3), 299-310 (2001)

Deng, Y., Blöte, H.W.J.: Monte Carlo study of the site-percolation model in two and three dimensions. Phys. Rev. E 72(1), 016126 (2005)

Durlofsky, L.J.: Use of higher moments for the description of upscaled, process independent relative permeabilities. SPE J. 2(4), 474-484 (1997)

Ekrann, S., Aasen, J.O.: Steady-state upscaling. Transp. Porous Media 41(3), 245-262 (2000)

Grimmett, G.: What is Percolation?. In: Percolation. Springer, Berlin, pp. 1-31 (1999)

Guzman, R.E., Giordano, D., Fayers, F.J., Godi, A., Aziz, K.: Evaluation of dynamic pseudofunctions for reservoir simulation. SPE J. 4(1), 37-46 (1999)

Hilden, S.T., Berg, C.F.: An analysis of unsteady flooding processes: varying force balance and the applicability of steady-state upscaling. Transp. Porous Media 115(1), 125-152 (2016)

Honarpour, M., Mahmood, S.M.: Relative-permeability measurements: an overview. J. Petrol. Technol. 40(8), 963-966 (1988)

Hunt, A., Ewing, R., Ghanbarian, B.: Percolation Theory for Flow in Porous Media, 880th edn. Springer, Berlin (2014)

Ioannidis, M.A., Chatzis, I., Sudicky, E.A.: The effect of spatial correlations on the accessibility characteristics of three-dimensional cubic networks as related to drainage displacements in porous media. Water Resour. Res. 29(6), 1777-1785 (1993)

Ioannidis, M.A., Chatzis, I., Dullin, A.L.: Macroscopic percolation model of immiscible displacement: effects of buoyancy and spatial structure. Water Resour. Res. 32(11), 3297-3310 (1996)

Jacks, H.H., Smith, O.J.E., Mattax, C.C.: The modeling of a three-dimensional reservoir with a twodimensional reservoir simulator-the use of dynamic pseudo functions. SPE J. 13(3), 175-185 (1973) 
Jacobsen, J.L.: Critical points of Potts and $\mathrm{O}(\mathrm{N})$ models from eigenvalue identities in periodic temperley-lieb algebras. J. Phys. A Math. Theor. 48(45), 454003-454033 (2015)

Johnson, E.F., Bossler, D.P., Bossler, V.O.N.: Calculation of relative permeability from displacement experiments. Trans. AIME 207, 370-372 (1959)

Jones, S.C., Roszelle, W.O.: Graphical techniques for determining relative permeability from displacement experiments. J. Petrol. Technol. 30(5), 807-817 (1978)

Jonoud, S., Jackson, M.D.: New criteria for the validity of steady-state upscaling. Transp. Porous Media 71(1), 53-73 (2008a)

Jonoud, S., Jackson, M.D.: Validity of steady-state upscaling techniques. SPE J. 11(2), 405-416 (2008b)

Juanes, R., Spiteri, E.J., Orr Jr., F.M., Blunt, M.J.: Impact of relative permeability hysteresis on geological $\mathrm{CO}_{2}$ storage. Water Resour. Res. 42, 1-13 (2006)

King, P.R.: The use of renormalization for calculating effective permeability. Transp. Porous Media 4(1), 37-58 (1989)

King, P.R., Muggeridge, A.H., Price, W.G.: Renormalization calculations of immiscible flow. Transp. Porous Media 12(3), 237-260 (1993)

Kueper, B.H., McWhorter, D.B.: The use of macroscopic percolation theory to construct large-scale capillary pressure curves. Water Resour. Res. 28(9), 2425-2436 (1992)

Kumar, A.T.A., Jerauld, G.R.: Impacts of scale-up on fluid flow from plug to Gridblock scale in reservoir rock. In: SPE/DOE Tenth Symposium on Improved Oil Recovery, SPE-35452-MS, pp. 1-16 (1996)

Kyte, J.R., Berry, D.W.: New pseudo functions to control numerical dispersion. SPE J. 15(4), 269-276 (1975)

Lee, M.J.: Complementary algorithms for graphs and percolation. Phys. Rev. E 76(2), 027702 (2007)

Leverett, M.: Capillary behavior in porous solids. Trans. AIME 141(1), 152-169 (1941)

Lohne, A., Virnovsky, G.A., Durlofsky, L.J.: Two-stage upscaling of two-phase flow: from core to simulation scale. SPE J. 11(3), 304-316 (2006)

Muskat, M.: The flow of fluids through porous media. J. Appl. Phys. 8(4), 274-282 (1937)

Newman, M.E.J., Ziff, R.M.: Efficient Monte Carlo algorithm and high-precision results for percolation. Phys. Rev. Lett. 85(19), 4104-4107 (2000)

Odsæter, L.H., Berg, C.F., Rustad, A.B.: Rate dependency in steady-state upscaling. Transp. Porous Media 110(3), 565-589 (2015)

Osoba, J.S., Richardson, J.G., Kerver, J.K., Hafford, J.A., Blair, P.M.: Laboratory measurements of relative permeability. J. Petrol. Technol. 3(2), 47-56 (1951)

Pickup, G.E., Stephen, K.D.: Steady-state scale-up methods. In: 6th European Conference on the Mathematics of Oil Recovery, Peebles, Scotland, pp. 8-11 (1998)

Pickup, G.E., Sorbie, K.S.: The scaleup of two-phase flow in porous media using phase permeability tensors. SPE J. 1(4), 369-381 (1996)

Richardson, J.G., Kerver, J.K., Hafford, J.A., Osoba, J.S.: Laboratory determination of relative permeability. J. Petrol. Technol. 4(8), 187-196 (1952)

Saad, N., Cullick, A.S., Honarpour, M.M.: Effective relative permeability in scale-up and simulation. In: Proceedings of SPE Rocky Mountain Regional/Low-Permeability Reservoirs Symposium, SPE-29592, pp. 451-464 (1995)

Saadatpoor, E., Bryant, S.L., Sepehrnoori, K.: New trapping mechanism in carbon sequestration. Transp. Porous Media 82(1), 3-17 (2010)

Škvor, J., Nezbeda, I.: Percolation threshold parameters of fluids. Phys. Rev. E 79(4), 041141 (2009)

Stephen, K.D., Pickup, G.E.: Steady state solutions of immiscible two-phase flow in strongly heterogeneous media: implications for upscaling. In: SPE Reservoir Simulation Symposium, SPE-51912-MS, pp. 1-2 (1999)

Stephen, K.D., Pickup, G.E., Sorbie, K.S.: The local analysis of changing force balances in immiscible incompressible two-phase flow. Transp. Porous Media 45(1), 63-88 (2001)

Stone, H.L.: Rigorous black oil pseudo functions. In: SPE Reservoir Simulation Symposium, SPE-21207, pp. 1-12 (1991)

Valvatne, P.H., Blunt, M.J.: Predictive pore-scale modeling of two-phase flow in mixed wet media. Water Resour. Res. 40(7), 1-20 (2004)

Virnovsky, G.A., Friis, H.A., Lohne, A.: A steady-state upscaling approach for immiscible two-phase flow. Transp. Porous Media 54(2), 167-192 (2004)

Wallstrom, T.C., Christie, M.A., Durlofsky, L.J., Sharp, D.H.: Effective flux boundary conditions for upscaling porous media equations. Transp. Porous Media 46(2), 139-153 (2002)

West, D.B.: Introduction to Graph Theory, 2nd edn. Prentice hall, Upper Saddle River (2001)

Xu, X., Wang, J., Lv, J.P., Deng, Y.: Simultaneous analysis of three-dimensional percolation models. Front. Phys. 9(1), 113-119 (2014) 
Yortsos, Y.C., Satik, C., Salin, D., Bacri, J.C.: Large-scale averaging of drainage at local capillary control. In: Proceedings of SPE Annual Fall Meeting, SPE-22592, pp. 55-70 (1991)

Yortsos, Y.C., Satik, C., Bacri, J.C., Salin, D.: Large-scale percolation theory of drainage. Transp. Porous Media 10(2), 171-195 (1993) 